ANNALES

POLONICI MATHEMATICI

LXXVI.3 (2001)

\title{
Une nouvelle version du théorème d'extension de Hartogs pour les applications séparément holomorphes entre espaces analytiques
}

\author{
par Omar Alehyane (El Jadida) et Ahmed Zeriahi (Toulouse)
}

\begin{abstract}
This paper is concerned with the problem of extension of separately holomorphic mappings defined on a "generalized cross" of a product of complex analytic spaces with values in a complex analytic space.

The crosses considered here are inscribed in Borel rectangles (of a product of two complex analytic spaces) which are not necessarily open but are non-pluripolar and can be quite small from the topological point of view.

Our first main result says that the singular set of a given separately holomorphic mapping defined on such a cross is quite small from the pluripotential point of view in the product space in the sense that each of its projections is pluripolar.

Then for some special crosses, we deduce more precise results on the extension of separately holomorphic mappings on such crosses, giving generalizations of all the main results obtained earlier by various authors in this direction.
\end{abstract}

1. Introduction. Le but essentiel de cet article est de donner une nouvelle version du célèbre théorème de Hartogs sur l'analyticité des fonctions séparément holomorphes [Ha]. Ce théorème a fait l'objet de nombreux travaux dont il est difficile de faire la liste exhaustive. Nous nous contenterons donc ici d'en citer les principaux.

Après Hartogs, ce fût sans doute l'école japonaise, avec notamment I. Shimoda [Shim] et T. Terada [Te], qui a initié le problème de l'holomorphie des fonctions séparément holomorphes sur un produit d'ouverts en affaiblissant les hypothèses sur l'un des facteurs, obtenant ainsi une généralisation conséquente du théorème de Hartogs.

Des extensions significatives du théorème de Terada ont été obtenues par la suite par J. Siciak (cf. [Si1], [Si2]). Il faut observer que Siciak fût le premier à donner au théorème de Hartogs une formulation générale en terme de prolongement de fonctions séparément holomorphes sur des "croix" d'un

2000 Mathematics Subject Classification: 31C10, 32D15, 32H02.

Key words and phrases: separately holomorphic, pluripolar, Hartogs theorem. 
espace hermitien produit et à obtenir les premiers résultats substantiels sur le sujet (cf. [Si1], [Si2]).

De plus il en a donné des applications à la détermination des enveloppes d'holomorphie de certains "domaines croisés" en utilisant les fonctions extrémales relatives qui sont l'analogue pluridimensionnel des mesures harmoniques en une dimension.

Les résultats de Siciak ont ensuite été généralisé par V. P. Zahariuta aux fonctions séparément holomorphes sur des "croix" d'un produit de variétés de Stein (cf. [Za2]) et des versions plus générales ont été obtenues plus tard par Nguyen Thanh Van et le second auteur avec des preuves plus simples (cf. [Ng-Ze2], [Ng-Ze3]).

Alors que Siciak avait essentiellement fait usage de la méthode d'interpolation des fonctions analytiques de plusieurs variables complexes, Zahariuta avait utilisé la méthode abstraite des échelles hilbertiennes qui se généralise facilement aux fonctions holomorphes définies sur des variétés de Stein (cf. [Za1], [Za2]).

Pour étendre les théorèmes de Siciak et Zahariuta, Nguyen Thanh Van et le second auteur avaient utilisé des développements en série dans une base doublement orthogonale de fonctions holomorphes, construite suivant une méthode classique dûe à S. Bergman [Ber], qui est en fait une version plus simple et plus constructive de la technique abstraite des échelles hilbertiennes développée par Zahariuta [Za1].

Cette méthode avait également été utilisée indépendamment par Borchers, qui ne semblait pas connaître la littérature sur le sujet, pour déterminer les enveloppes d'holomorphie de certains domaines croisés avec des motivations provenant de la physique théorique (cf. [Bo]).

Utilisant essentiellement la même méthode que Siciak, B. Shiffman fût le premier à étendre partiellement certains résultats de Siciak et ceux de Terada aux applications séparément holomorphes à valeurs dans un espace analytique complexe (cf. [Shif2], [Shif3]).

Reprenant la méthode de [Ng-Ze2], [Ng-Ze3] et utilisant une idée de [Shif2], [Shif3], O. Alehyane a pu généraliser certains résultats de [Ng-Ze2], [Ng-Ze3], [Shif3] aux applications séparément holomorphes à valeurs dans un espace analytique complexe vérifiant la propriété de prolongement de Hartogs (cf. [Al1]).

Par ailleurs, Saint Raymond [Ra] puis Siciak [Si4] ont mis en évidence la nature "doublement pluripolaire" de l'ensemble singulier d'une fonction séparément analytique au sens réel sur un domaine d'un produit d'espaces euclidiens et ont obtenu une caractérisation élégante de ces ensembles singuliers.

Bien que la démonstration de Siciak de la condition nécessaire de ce résultat repose sur une version très simple de son théorème de prolongement 
des fonctions séparément holomorphes sur une croix convenable (cf. [Si1]), il n'en demeure pas moins que le lien entre ces deux résultats n'était pas direct.

Notre objectif ici est de donner un nouveau théorème d'extension de type Hartogs aussi général que possible avec des hypothèses aussi simples que possible, impliquant de manière directe tous les résultats importants cités ci-dessus et permettant en particulier de clarifier le lien entre le cas réel et le cas complexe en unifiant les deux points de vue.

En effet, nous considérons une croix inscrite dans un rectangle borelien non-pluripolaire (contenu dans un produit d'espaces analytiques complexes) d'un type assez général et une application séparément holomorphe définie sur cette croix à valeurs dans un espace analytique complexe quelconque. Nous montrons qu'alors l'ensemble singulier d'une telle application est "doublement pluripolaire".

Cette nouvelle formulation du théorème d'extension de Hartogs nous permet en particulier d'appliquer nos résultats à des fonctions séparément analytiques au sens réel et d'obtenir ainsi d'une façon directe une généralisation significative de la condition nécessaire dans le théorème de Saint Raymond et Siciak.

Enfin nous en déduisons plusieurs résultats nouveaux qui généralisent tous les résultats essentiels cités ci-dessus.

2. Préliminaires et énoncés des résultats. Ici nous rappelons les notions de base et les résultats préliminaires qui serviront dans la suite, puis nous énoncerons les résultats principaux obtenus dans ce travail.

2.1. Définitions et résultats préliminaires. Dans toute la suite les espaces analytiques complexes considérés seront toujours supposés réduits, irréductibles et de dimension finie. Si $X$ est un espace analytique complexe, on désignera par $X_{\text {reg }}$ (resp. $X_{\text {sing }}$ ) la variété analytique complexe des points réguliers de $X$ (resp. l'ensemble singulier de $X$, qui est un sous-ensemble analytique de $X$ de codimension au moins 1$)$.

Rappelons qu'une fonction $u: X \rightarrow[-\infty,+\infty[$ est dite fortement plurisousharmonique sur $X$ si au voisinage de chaque point de $X$, dans un plongement local de $X$ dans un espace hermitien, $u$ est la restriction d'une plurisousharmonique.

La fonction $u$ est dite (faiblement) plurisousharmonique sur $X$ si $u$ est plurisousharmonique sur la variété complexe $X_{\text {reg }}$ et localement majorée sur $X$. Dans cette définition, on n'impose pas que $u$ soit semi-continue supérieurement. Pour remédier à cet inconvénient, à chaque fonction $u$ plurisousharmonique sur $X$ on associera une fonction semi-continue supérieurement sur $X$ en posant

$$
u^{*}(x)=\limsup _{y \rightarrow x, y \in X_{\mathrm{reg}}} u(y), \quad x \in X .
$$


La fonction $u^{*}$ sera appelée la régularisée semi-continue supérieurement de $u$ sur $X$. Notons que si $u$ est fortement plurisousharmonique sur $X$ alors $u=u^{*}$ est (faiblement) plurisousharmonique sur $X$. La réciproque n'a pas lieu en général (cf. [De], [Ze2]).

Une version du lemme de Hartogs pour les fonctions plurisousharmoniques sur les espaces analytiques complexes est donnée dans [Ze2].

Dans toute la suite on notera $P(X)$ le cône des fonctions (faiblement) plurisousharmoniques sur $X$, non identiquement égales à $-\infty$.

L'opérateur de Monge-Ampère complexe $\left(d d^{c} u\right)^{m}$ est bien défini pour une fonction $u$ plurisousharmonique localement bornée sur la variété $X_{\text {reg }}$ (cf. [Bed]). Il en résulte que si $u \in P(X) \cap L_{\text {loc }}^{\infty}(X)$, alors $\left(d d^{c} u\right)^{m}$ est un courant positif fermé de bidegré $(m, m)$ sur $X_{\text {reg }}$. On peut prolonger ce courant par 0 sur $X_{\text {sing }}$ (cf. [Bed]).

À l'aide de cet opérateur, on peut définir une capacité de la façon suivante. Rappelons tout d'abord qu'un ouvert $U \subset X$ est dit hyperconvexe s'il admet une fonction d'exhaustion plurisousharmonique et bornée.

Soient $U \subset X$ un domaine hyperconvexe et $K \subset U$ un compact. On définit suivant Bedford et Taylor [Bd-Ta] la capacité du "condensateur" $(K, U)$ par la formule

$$
\operatorname{Cap}(K ; U):=\sup \left\{\int_{K}\left(d d^{c} u\right)^{m}: u \in P(U), 0 \leq u \leq 1\right\}
$$

et on étend cette définition de façon naturelle à tous les ensembles en définissant la capacité extérieure Cap* (cf. [Bd-Ta]). Alors la capacité ainsi obtenue est une capacité au sens de Choquet qui caractérise les ensembles pluripolaires au sens où un sous-ensemble $E \subset U$ est pluripolaire si et seulement si $\operatorname{Cap}^{*}(E ; U)=0$ (cf. [Bd-Ta]).

Pour un domaine $U \subset X$ et un ensemble non vide $E \subset U$, on définit suivant Siciak ([Si2], [Si3]) la fonction extrémale relative associée au couple $(E, U)$ par la formule suivante :

$$
u_{E, U}(x)=\sup \{u(x): u \in P(U), u \leq 0 \text { sur } E, u \leq 1 \text { sur } U\} .
$$

On note $\omega(\cdot ; E ; U)=u_{E, U}^{*}$ la régularisée s.c.s. de $u_{E, U}$ qui est alors une fonction plurisousharmonique sur $U$ qui vérifie l'équation de Monge-Ampère complexe (cf. [Be-Ta], [Bed]) :

$$
\left(d d^{c} \omega(\cdot ; E ; U)\right)^{m}=0 \quad \text { sur } U \backslash \bar{E} .
$$

Cette fonction est aussi appelée la $P$-mesure de $E$ relative à $U$, par analogie à la mesure harmonique en une variable complexe (cf. [Sa]).

Pour tout $\alpha \in] 0,1[$, on définit l'ouvert de sous-niveau $\alpha$ de la fonction $\omega(. ; E ; U)$ par la formule suivante :

$$
U(E, \alpha):=\{x \in U: \omega(x ; E ; U)<\alpha\} .
$$


On dira que $E \subset U$ est $P(U)$-régulier au point $a \in \bar{E} \cap U$ si $\omega(a ; E ; U)=0$. L'ensemble $E$ est dit plurirégulier au point $a \in \bar{E} \cap U$ si pour tout voisinage ouvert $D$ de $a, E \cap D$ est $P(D)$-régulier au point $a$, ce qui équivaut à la propriété suivante :

$$
\omega(a ; E \cap D ; D)=0 \quad \text { pour tout } D \in \mathfrak{V}(a)
$$

où $\mathfrak{V}(a)$ est un système fondamental de voisinages ouverts du point $a$. On désigne par $E^{*}=E_{U}^{*}$ l'ensemble des points $a \in \bar{E} \cap U$ tels que $E$ soit plurirégulier au point $a$. Si $E$ est non-pluripolaire, alors d'après Bedford et Taylor ([Be-Ta], [Bed]) l'ensemble $E^{*}$ est un ensemble non-pluripolaire de type $\mathcal{G}_{\delta}$ et $E \backslash E^{*}$ est pluripolaire. De plus on vérifie facilement que $\left(E^{*}\right)^{*}$ $=E^{*}$.

Rappelons également que si $U$ est hyperconvexe alors pour tout ensemble $E \subset U$ et toute suite exhaustive $\left(U_{j}\right)$ d'ouverts relativement compacts de $U$ on a la propriété de stabilité (par rapport à $U$ ) suivante (cf. [Kl]) :

$$
\omega(\cdot ; E ; U)=\lim _{j \rightarrow+\infty} \omega\left(\cdot ; E \cap U_{j} ; U_{j}\right) .
$$

En général cette propriété de stabilité de la fonction extrémale n'est pas satisfaite si $U$ est seulement pseudoconvexe (cf. [Al-He]).

Il est donc naturel dans notre contexte de définir une nouvelle fonction associée à $(E, U)$ en posant

$$
\widetilde{\omega}(\cdot ; E ; U):=\lim _{j \rightarrow+\infty} \omega\left(\cdot ; E \cap U_{j} ; U_{j}\right)
$$

où $\left(U_{j}\right)$ est une suite exhaustive d'ouverts relativement compacts de $U$. Il est facile de voir que cette limite est indépendante de la suite exhaustive choisie (cf. [Za2]).

On vérifie facilement que la nouvelle fonction possède la propriété suivante, dite propriété de conservation de la $P$-mesure :

$$
\widetilde{\omega}(\cdot ; E \cup S ; U) \equiv \widetilde{\omega}(\cdot ; E ; U)
$$

pour tout sous-ensemble pluripolaire $S \subset U$. Il en résulte que si $E \subset U$ est non-pluripolaire, on a

$$
\widetilde{\omega}\left(\cdot ; E^{*} ; U\right) \equiv \widetilde{\omega}(\cdot ; E ; U) .
$$

À partir de cette propriété, on démontre facilement la propriété de stabilité pour la fonction $\widetilde{\omega}(\cdot ; E ; U)$ par rapport à la fois à $U$ et à $E$. C'est pourquoi on l'appellera la fonction plurisousharmonique extrémale (relative) stable de $(E, U)$. On a toujours $\omega(\cdot ; E ; U) \leq \widetilde{\omega}(\cdot ; E ; U)$ avec égalité si $U$ est relativement compact ou hyperconvexe (cf. [Kl]). On notera alors

$$
\widetilde{U}(E, \alpha):=\{x \in U: \widetilde{\omega}(x ; E ; U)<\alpha\} .
$$

Rappelons également que si $U$ est un domaine hyperconvexe et $E \subset U$ est un compact non-pluripolaire, alors la mesure de Monge-Ampère relative 
$\mu=\mu_{E, U}:=\left(d d^{c} \omega(\cdot ; E ; U)\right)^{n}$ possède la propriété fondamentale suivante (cf. [Ng-Ze1], [Ze2]) : pour tout ensemble borelien $A \subset E$ tel que $\mu(A)=\mu(E)$, on a

$$
\omega(\cdot ; A ; U) \equiv \omega(\cdot ; E ; U) .
$$

Pour simplicité, si $E \subset X$ est un sous-ensemble non-pluripolaire de $X$, on dira qu'un ouvert $U^{\prime} \subset X$ est un voisinage consistant de $E$ si $U^{\prime}$ est un voisinage ouvert de $E$ dont toute composante connexe rencontre $E^{*}$, ce qui revient à dire que toute composante connexe de $U^{\prime}$ rencontre $E$ suivant un ensemble non-pluripolaire. L'intérêt de cette notion réside dans la propriété suivante : si $f$ et $g$ sont deux applications holomorphes sur $U^{\prime}$ telles que $f=g$ sur $E$, alors on a $f=g$ sur $U^{\prime}$. On fera référence à ce résultat comme étant le principe du prolongement analytique généralisé.

On dira qu'un sous-ensemble $E^{\prime} \subset E^{*}$ est quasi-égal à $E$ dans $X$ si l'ensemble $E \backslash E^{\prime}$ est pluripolaire dans $X$.

On peut ainsi montrer que si $E \subset U$ est non-pluripolaire, alors $E^{*}$ est quasi-égal à $E$ et que l'ouvert $\widetilde{U}(\alpha, E)$ est un voisinage consistant de $E^{*}$ (cf. [Ng-Ze3]).

On dira qu'une partie $S \subset X \times Y$ est doublement pluripolaire dans $X \times Y$ si les projections $\pi_{X}(S) \subset X$ et $\pi_{Y}(S) \subset Y$ sont pluripolaires dans $X$ et $Y$ respectivement.

Nous aurons besoin de la propriété de prolongement de Hartogs (PPH) introduite par Shiffman [Shif1]. Soit $p \geq 2$ un entier. Pour $r \in] 0,1[$, on note $H_{p}(r)$ la figure de Hartogs en dimension $p$ définie par

$$
H_{p}(r)=\left\{\left(z^{\prime}, z_{p}\right) \in \Delta^{p}:\left\|z^{\prime}\right\|<r \text { ou }\left|z_{p}\right|>1-r\right\}
$$

où $\Delta^{p}$ désigne le polydisque unité de $\mathbb{C}^{p}$ et $z^{\prime}=\left(z_{1}, \ldots, z_{p-1}\right),\left\|z^{\prime}\right\|=$ $\max _{1 \leq j \leq p-1}\left|z_{j}\right|$.

DÉfinition 2.1.1. On dira qu'un espace analytique complexe $Z$ possède la propriété de prolongement de Hartogs ( $\mathrm{PPH})$ en dimension $p$ si toute application holomorphe de $H_{p}(r)$ à valeurs dans $Z$ se prolonge en une application holomorphe de $\Delta^{p}$ dans $Z$.

S. Ivashkovich [Iv1] a démontré que si l'espace $Z$ possède la propriété $(\mathrm{PPH})$ en dimension 2 alors il la possède en toute dimension $p \geq 2$. Nous dirons simplement que $Z$ possède la propriété (PPH). La classe des espaces analytiques complexes possédant la propriété (PPH) contient les groupes de Lie complexes [Ad-Su-Yo], les espaces tauts [W], les variétés hermitiennes à courbures sectionnelles holomorphes négatives [Shif1] et les variétés kähleriennes holomorphiquement convexes ne contenant pas de courbe rationnelle [Iv1].

Voici une caractérisation importante de cette classe d'espaces analytiques complexes que nous utiliserons ultérieurement. 
ThÉORÈme 2.1.2 ([Shif1]). Un espace analytique complexe $Z$ possède la propriété $(P P H)$ (en dimension $p$ ) si et seulement si pour tout domaine $D$ d'une variété de Stein quelconque $N$, toute application holomorphe de $D$ dans $Z$ se prolonge en une application holomorphe de l'enveloppe d'holomorphie $\widehat{D}$ de $D$ dans $Z$.

2.2. Énoncés des résultats principaux. Nous aurons besoin de quelques définitions pour poser le problème général de l'extension des applications séparément holomorphes et énoncer les principaux résultats obtenus.

Soient $X, Y$ et $Z$ des espaces analytiques complexes quelconques. Soient $K \subset X, L \subset Y$ des ensembles boreliens connexes non-pluripolaires, et $E \subset K$, $F \subset L$ des sous-ensembles non-pluripolaires dans $X$ et $Y$ respectivement. L'ensemble $W:=(K \times F) \cup(E \times L)$ sera appelé la croix de $X \times Y$ inscrite dans le rectangle borelien $K \times L$ et centrée sur le rectangle $E \times F$. Lorsqu'il y a risque de confusion, on notera de façon plus précise $W=W(E \times F ; K \times L)$.

On dira qu'une application $f: W(E \times F ; K \times L) \rightarrow Z$ est séparément holomorphe sur $W$ si elle vérifie les deux propriétés suivantes :

1. pour tout $x \in E$, l'application partielle $f(x, \cdot): L \rightarrow Z$ se prolonge en une application holomorphe d'un voisinage ouvert de $L$ dans $Y$ (dépendant éventuellement de $x$ ) à valeurs dans $Z$,

2. pour tout $y \in F$, l'application partielle $f(\cdot, y): K \rightarrow Z$ se prolonge en une application holomorphe d'un voisinage ouvert de $K$ dans $X$ (dépendant éventuellement de $y$ ) à valeurs dans $Z$.

Lorsque $Z=\mathbb{C}$, on parlera simplement de fonction (séparément) holomorphe plutôt que d'application.

Définissons maintenant les différents types d'ensembles boreliens $K$ et $L$ que nous aurons à considérer. Rappelons qu'un sous-ensemble borelien $K \subset X$ est dit de type $\mathcal{F}_{\sigma}$ s'il peut s'écrire comme réunion dénombrable d'ensembles fermés de $X$, que l'on peut toujours choisir compacts. Un sousensemble $K \subset X$ est dit de type $\mathcal{G}_{\delta}$ dans $X$ s'il peut s'écrire comme une intersection dénombrable d'ouverts de $X$.

On dira dans la suite qu'un sous-ensemble $K \subset X$ est de type $\mathcal{G}_{\delta}^{s}$ s'il existe une suite décroissante $\left(D_{j}\right)_{j}$ d'ouverts de $X$ contenant $K$ qui converge vers $K$ au sens suivant : pour tout ouvert $D$ contenant $K$, il existe un indice $j$ tel que $D_{j} \subset D$. Il est clair que tout sous-ensemble qui est réunion d'un compact et d'un ouvert de $X$ est de type $\mathcal{G}_{\delta}^{s}$.

Récemment W. Jarnicki [Ja] nous a fait remarquer qu'en fait tout ensemble de type $\mathcal{G}_{\delta}^{s}$ est réunion d'un ouvert et d'un compact.

On notera $W^{*}:=\left(K \times F^{*}\right) \cup\left(E^{*} \times L\right)$ la partie dite régulière de $W$.

Lorsque $W=W(E \times F ; U \times V)$ est une croix inscrite dans un rectangle ouvert $U \times V \subset X \times Y$, on posera 


$$
\widetilde{W}=\{(x, y) \in U \times V: \widetilde{\omega}(x ; E ; U)+\widetilde{\omega}(y ; F ; V)<1\} .
$$

Il faut observer que $W^{*} \subset \widetilde{W}$.

Un ouvert $\Omega \subset X \times Y$ est dit admissible pour l'extension des applications séparément holomorphes sur $W$ si toute composante connexe de $\Omega$ rencontre $W^{*}$.

Soit $f: W:=(K \times F) \cup(E \times L) \rightarrow Z$ une application. On dira alors que $f$ s'étend holomorphiquement à un ouvert admissible $\Omega$ s'il existe une application holomorphe $h$ de $\Omega$ dans $Z$ telle que $h=f$ sur $\Omega \cap W$. Si une telle extension holomorphe au même ouvert $\Omega$ est possible pour toutes les fonctions séparéments holomorphes sur $W$, on dira que $\Omega$ est un ouvert d'extension holomorphe de $W$.

Si $(a, b) \in X \times Y$, on dira que $f$ s'étend holomorphiquement au voisinage $d u$ point $(a, b)$ s'il existe un domaine $\omega=\omega_{a, b}$ de $X \times Y$ voisinage du point $(a, b)$ tel que $\omega \cap W^{*} \neq \emptyset$ et tel que $f$ s'étende holomorphiquement à $\omega$.

On désignera par $\Omega(f)$ l'ensemble des points de $X \times Y$ au voisinage desquels l'application $f$ s'étend holomorphiquement. Il est clair que $\Omega(f)$ est un ouvert admissible pour l'extension holomorphe des fonctions séparément holomorphes sur $W$. En général, l'application $f$ ne s'étend pas en une application holomorphe sur l'ouvert $\Omega(f)$ tout entier à valeurs dans $Z$, même dans le cas où $Z=\mathbb{C}$. En effet, considérons la fonction définie par $f(x, y):=$ $\left(x^{2}+y^{2}\right)^{1 / 2}$ pour $\left.\left.\left.\left.(x, y) \in W:=(] 0,1\right] \times \mathbb{R}^{+}\right) \cup\left(\mathbb{R}^{+} \times\right] 0,1\right]\right) \subset \mathbb{C} \times \mathbb{C}$. Il est clair que $f: W \rightarrow \mathbb{C}$ est une fonction séparément holomorphe sur $W$, que $\Omega(f)=\mathbb{C}^{2} \backslash\left\{(x, y) \in \mathbb{C}^{2}: x^{2}+y^{2}=0\right\}$ et que $S(f, W)=\{(0,0)\}$. Par contre la fonction $f$ ne s'étend pas en une fonction holomorphe sur l'ouvert $\Omega(f)$. Ici le prolongement naturel est une fonction analytique multiforme.

Plusieurs questions naturelles se posent alors dans ce contexte général.

Q.1 : Étant donnée une application $f: W \rightarrow Z$ séparément holomorphe sur $W$, que peut-on dire de l'ensemble singulier de $f$ relativement à $W$ defini $\operatorname{par} S(f, W):=W^{*} \backslash \Omega(f) ?$

Q.2 : L'ouvert $\Omega(f)$ contient-il un voisinage ouvert admissible de $W^{*}$ dans $X \times Y$ auquel $f$ s'étend holomorphiquement?

Q.3 : Soient $X$ et $Y$ des variétés de Stein, $U \subset X$ et $V \subset Y$ des domaines et $E \subset U, F \subset V$ des ensembles non-pluripolaires. Déterminer l'enveloppe d'holomorphie de $W$ au sens de l'extension holomorphe des fonctions séparément holomorphes sur la croix $W:=W(E \times F ; U \times V)$, à savoir la variété de Stein admissible $H(W)$ contenant $W^{*}$ à laquelle toutes les fonctions séparément holomorphes sur $W$ s'étendent holomorphiquement.

Q.4 : Dans les hypothèses de $\mathbf{Q . 3}$, caractériser les espaces analytiques complexes $Z$ pour lesquels toute application séparément holomorphe sur $W$ s'étend holomorphiquement à $H(W)$. 
Avant d'énoncer les résultats essentiels de ce travail, donnons un aperçu chronologique succinct des principaux résultats obtenus antérieurement sur le sujet.

Il semble que I. Shimoda [Shim] et T. Terada [Te] fûrent parmi les premiers à obtenir des résultats importants dans cette direction en considérant des fonctions séparément holomorphes sur des ensembles de la forme $W=W(E \times V ; U \times V)$, i.e. une croix telle que $F=V \subset \mathbb{C}^{m}$ et $E \subset U \subset \mathbb{C}^{n}$ soit un ensemble non-pluripolaire. Ils ont démontré que de telles fonctions sont nécessairement holomorphes sur $U \times V$.

Lorsque $X=\mathbb{C}^{m}, Y=\mathbb{C}^{n}, Z=\mathbb{C}$ et $E \subset U, F \subset V$ sont des compacts, J. Siciak a été le premier à formuler la question $\mathbf{Q . 3}$ pour une croix inscrite dans un rectangle ouvert et à démontrer que, pour certains types de domaines $U$ et $V$ et pour des compacts réguliers $E \subset U$ et $F \subset V$, l'enveloppe d'holomorphie de $W=W(E \times F ; U \times V)$ est $H(W)=\widetilde{W}$ (cf. [Si1]-[Si3]).

Par la suite, V. P. Zahariuta a généralisé les résultats de Siciak au cas où $X$ et $Y$ sont des variétés de Stein, $Z=\mathbb{C}$ et $U \subset X, V \subset Y$ sont des domaines pseudoconvexes et $E \subset U, F \subset V$ sont des compacts réguliers (cf. [Za2]). Plus tard ces résultats ont été généralisés par Nguyen Thanh Van et le second auteur au cas où $U \subset \mathbb{C}^{m}$ et $V \subset \mathbb{C}^{n}$ sont des domaines bornés ou hyperconvexes et les ensembles $E \subset U$ et $F \subset V$ sont seulement nonpluripolaires avec cependant une hypothèse technique supplémentaire sur l'un des facteurs, à savoir que par exemple $E$ est borelien et $U$ pseudoconvexe (cf. [Ng-Ze2], [Ng-Ze3]).

Le cas des applications séparément holomorphes sur des rectangles mixtes à valeurs dans un espace analytique quelconque a été étudié plus tard par B. Shiffman (cf. [Shif1], [Shif2]). Reprenant des méthodes analogues à celles de [Ng-Ze2], [Ng-Ze3] et de [Shif1], [Shif2], O. Alehyane a généralisé ensuite les résultats de [Ng-Ze2], [Ng-Ze3] au cas des applications séparément holomorphes à valeurs dans un espace analytique complexe ayant la propriété de prolongement de Hartogs (PPH), en supprimant l'hypothèse technique sur l'un des facteurs.

Par ailleurs, Saint Raymond ([Ra]) puis J. Siciak ([Si4]) ont obtenu un résultat remarquable sur la caractérisation de l'ensemble singulier des applications séparément analytiques au sens réel sur des ouverts de $\mathbb{R}^{m} \times \mathbb{R}^{n}$. Ce résultat a ensuite été généralisé et précisé par $\mathrm{Z}$. Błocki [Bł].

Nous pouvons maintenant énoncer les résultats principaux de ce travail.

ThÉORÈme 2.2.1. Soient $X, Y, Z$ des espaces analytiques complexes, $K \subset X, L \subset Y$ des ensembles boreliens connexes non-pluripolaires de type $\mathcal{F}_{\sigma}$ et $E \subset K, F \subset L$ des ensembles non-pluripolaires quelconques. Soit $f: W:=(K \times F) \cup(E \times L) \rightarrow Z$ une application séparément holomorphe sur $W$. Alors il existe un ensemble borelien $E^{\prime} \subset E^{*}$ quasi-égal à $E$ dans 
$X$ et un ensemble borelien $F^{\prime} \subset F^{*}$ quasi-égal à $F$ dans $Y$ tels que $W^{\prime}:=$ $\left(K \times F^{\prime}\right) \cup\left(E^{\prime} \times L\right) \subset \Omega(f)$. En particulier, l'ensemble singulier $S(f, W):=$ $W^{*} \backslash \Omega(f)$ de $f$ est doublement pluripolaire dans $X \times Y$.

Ce théorème répond clairement à la question Q.1. L'exemple qui suit le théorème 2.2.2 montre que l'on ne peut pas faire mieux en général.

Dans le cas d'une application séparément holomorphe sur une croix $W$ inscrite dans un rectangle de type $\mathcal{G}_{\delta}^{s}$, on peut préciser le résultat précédent en déterminant un voisinage ouvert admissible de la croix $W^{\prime}$ donnée par le théorème précédent, auquel $f$ s'étend en une application holomorphe.

ThÉORÈme 2.2.2. Soient $X, Y, Z$ des espaces analytiques complexes et $K \subset X, L \subset Y$ des ensembles boreliens connexes non-pluripolaires de type $\mathcal{G}_{\delta}^{s}$. Soient $E \subset K, F \subset L$ des ensembles non-pluripolaires dans $X$ et $Y$ respectivement et $f: W:=(K \times F) \cup(E \times L) \rightarrow Z$ une application séparément holomorphe sur $W$. Alors il existe un ensemble borelien $E^{\prime} \subset E^{*}$ quasi-égal $\grave{a} E$ dans $X$ et un ensemble borelien $F^{\prime} \subset F^{*}$ quasi-égal à $F$ dans $Y$, et il existe un voisinage ouvert $\Omega$ de $W^{\prime}:=\left(K \times F^{\prime}\right) \cup\left(E^{\prime} \times L\right)$ dans $X \times Y$ et une application $\widetilde{f}$ holomorphe de $\Omega$ dans $Z$ tels que $\tilde{f}=f$ sur $\Omega \cap W$.

Donnons un exemple simple qui montre clairement que les théorèmes précédents ne peuvent guère être améliorés en géneral.

En effet, considérons l'application $f: \mathbb{C}^{2} \rightarrow \mathbb{P}_{1}$ définie par $f(z, w):=$ $\left[(z+w)^{2}:(z-w)^{2}\right]$ si $(z, w) \neq(0,0)$ et $f(0,0):=[1: 1]$. On vérifie facilement que $f$ est séparément holomorphe sur $\mathbb{C} \times \mathbb{C}$, holomorphe sur $\mathbb{C}^{2} \backslash\{(0,0)\}=\left(\mathbb{C} \times \mathbb{C}^{*}\right) \cup\left(\mathbb{C}^{*} \times \mathbb{C}\right)$ mais n'est pas continue en $(0,0)$. En particulier on a $S(f, W)=\{(0,0)\}$. Ainsi l'ensemble singulier considéré dans le théorème 2.2.1 est en général non vide et la croix $W^{\prime}$ fournie par le théorème 2.2 .2 n'est pas égale à $W^{*}$.

Remarquons que le même phénomène se produit lorsque $Z$ est un espace analytique contenant une courbe rationnelle.

Observons que si la croix $W$ est inscrite dans un rectange $K \times L$ de $\mathbb{R}^{m} \times \mathbb{R}^{n}$, plongé de façon naturelle dans $\mathbb{C}^{m} \times \mathbb{C}^{n}$, avec des hypothèses convenables, on obtient de façon immédiate à partir du théorème précédent une version plus générale et plus précise de la condition nécessaire du théorème de Saint Raymond [Ra] et Siciak [Si4].

Corollaire 2.2.3. Soient $K \subset \mathbb{R}^{m}, L \subset \mathbb{R}^{n}$ des ensembles boreliens connexes non-pluripolaires de type $\mathcal{F}_{\sigma}$ et $E \subset K, F \subset L$ des ensembles nonpluripolaires. Soit $f: W:=(K \times F) \cup(E \times L) \rightarrow \mathbb{C}$ une fonction séparément analytique au sens réel sur $W$. Alors l'ensemble singulier $S(f, W)$ de $f$ est doublement pluripolaire dans $\mathbb{C}^{m} \times \mathbb{C}^{n}$.

Si de plus $K \subset \mathbb{R}^{m}$ et $L \subset \mathbb{R}^{n}$ sont des ensembles de type $\mathcal{G}_{\delta}^{s}$, alors il existe un sous-ensemble borelien $E^{\prime} \subset E^{*}$ quasi-égal à $E$, un sous-ensemble 
borelien $F^{\prime} \subset F^{*}$ quasi-égal à $F$ et un voisinage ouvert $\Omega$ de $\left(E^{\prime} \times L\right) \cup$ $\left(K \times F^{\prime}\right)$ dans $\mathbb{C}^{m} \times \mathbb{C}^{n}$ tels que la fonction $f$ s'étende en une fonction holomorphe sur $\Omega$.

Enfin dans le cas où l'espace analytique complexe d'arrivée $Z$ possède la propriété de prolongement de Hartogs, ce qui exclut l'exemple précédent (cf. [Iv1]), on obtient des résultats beaucoup plus précis.

THÉORÈme 2.2.4. Soient $X$ et $Y$ des variétés analytiques complexes et $Z$ un espace analytique complexe possèdant la propriété de prolongement de Hartogs $(P P H)$. Soient $U \subset X, V \subset Y$ des domaines, $E \subset U, F \subset V$ des ensembles non-pluripolaires et $f: W:=(U \times F) \cup(E \times V) \rightarrow Z$ une application séparément holomorphe sur $W$. Alors on a les propriétés suivantes :

1) Si $X$ et $Y$ sont des variétés de Stein, l'application $f$ s'étend en une application holomorphe sur $\widetilde{W}$ à valeurs dans $Z$, autrement dit l'enveloppe d'holomorphie de $W$ au sens de l'extension des applications séparément holomorphes coïncide avec $\widetilde{W}$.

2) Dans le cas général, l'application f s'étend en une application holomorphe d'un voisinage ouvert $\Omega$ de $W^{*}$ à valeurs dans $Z$.

On en déduit un résultat intéressant du type "théorème de Terada" (cf. [Te]), qui constitue une généralisation d'un résultat de Shiffman ([Shif], Theorem 1) et du résultat principal de [Ku-Ha].

Corollaire 2.2.5. Soient $X$ et $Y$ des variétés analytiques complexes et $Z$ un espace analytique complexe possèdant la propriété de prolongement de Hartogs $(P P H)$. Soient $U \subset X$ un domaine, $L \subset Y$ un ensemble borelien connexe de type $\mathcal{G}_{\delta}^{s}$ et non-pluripolaire dans $Y$ et $E \subset U$ un ensemble nonpluripolaire dans $X$. Alors toute application $f: W=W(E \times L ; U \times L):=$ $U \times L \rightarrow Z$ séparément holomorphe sur $W$ s'étend en une application holomorphe d'un voisinage ouvert de $U \times L^{*}$ à valeurs dans $Z$.

$S i$ de plus $U$ est hyperconvexe, alors pour tout $\varepsilon>0$, il existe un sousensemble borelien $E^{\prime} \subset E$ tel que $\operatorname{Cap}^{*}\left(E \backslash E^{\prime} ; U\right)<\varepsilon$, et il existe un domaine $G$ voisinage de $L$ tels que $f$ s'étende holomorphiquement à un voisinage ouvert $\Omega$ de $\left(E^{\prime} \times G\right) \cup\left(U \times L^{*}\right)$.

Le théorème 2.2.4, ainsi qu'un analogue de ce théorème pour les applications séparément méromorphes, ont été obtenus tout d'abord par Alehyane dans le cas où $X$ et $Y$ sont des espaces hermitiens [Al2]. Par la suite, Ivashkovich [Iv2] a donné un analogue du théorème 2.2.2 pour les applications séparément méromorphes sur une croix inscrite dans un rectangle ouvert, avec des techniques différentes. Par ailleurs nos méthodes doivent permettre de généraliser les théorèmes précédents au cas des applications séparément méromorphes (cf. [Al2] pour des résultats partiels). 
Pour démontrer les résultats que nous venons d'énoncer, nous aurons besoin d'établir tout d'abord des théorèmes d'extensions du type Hartogs pour les fonctions séparément holomorphes. Dans ce cas les résultats que nous obtenons sont en fait plus précis et constituent des généralisations intéressantes de résultats obtenus notamment dans [Ng-Ze2], [Al1], [Shif1].

Les résultats concernant l'extension des fonctions séparément holomorphes seront énoncés et démontrés au paragraphe 3 . Ces résultats seront ensuite utilisés au paragraphe 4 pour démontrer les théorèmes d'extension pour les applications séparément holomorphes énoncés ci-dessus.

3. Extension de fonctions séparément holomorphes. Le but essentiel de ce paragraphe est de démontrer des théorèmes d'extension pour les fonctions séparément holomorphes sur une croix inscrite dans un rectangle ouvert. Cela se fera en plusieurs étapes. L'étape essentielle consiste à montrer que si $f$ est séparément holomorphe sur une telle croix $W$, alors elle se prolonge holomorphiquement à un voisinage ouvert de $W^{*}$.

3.1. Le système doublement orthogonal de Bergman. Notre méthode repose sur les développements en série suivant un système doublement orthogonal utilisé dans [Ng-Ze2], [Ng-Ze3]. Soient $V \Subset Y$ un domaine et $F \subset V$ un compact non-pluripolaire. On notera $H_{1}$ l'espace de Bergman des fonctions holomorphes sur $V$ dont le module est de carré intégrable sur $V$. Soit $H_{0}$ la fermeture de $H_{1}$ dans $L^{2}(F, d \mu), \mu$ étant la mesure de Monge-Ampère associée à $(F, V)$. On dira qu'un domaine $V \Subset Y$ est fortement hyperconvexe s'il existe un domaine pseudoconvexe $G \ni V$ et une fonction $\varphi: G \rightarrow$ ] $-\infty, 0[$ plurisousharmonique continue telle que $V=\{y \in G: \varphi(y)<0\}$. Le lemme suivant est classique et jouera un rôle fondamental (cf. [Ng-Ze2], [Ng-Ze3], [Ze3]).

Lemme 3.1.1. Soient $V \Subset Y$ un domaine fortement hyperconvexe et $F \subset V$ un compact non-pluripolaire. Alors il existe une base $\left(B_{j}\right)_{j \geq 1}$ doublement orthogonale dans $H_{1}$ et $H_{0}$ telle que

$$
\left\|B_{j}\right\|_{H_{0}}=1, \quad \forall j \geq 1, \quad \text { et } \quad\left\|B_{j}\right\|_{H_{1}}=\mu_{j} \nearrow+\infty .
$$

De plus pour tout $\varepsilon>0$, on a

$$
\begin{gathered}
\sum_{j=1}^{+\infty} \mu_{j}^{-\varepsilon}<+\infty, \\
\limsup _{j \rightarrow+\infty} \frac{\log \left|B_{j}(x)\right|}{\log \mu_{j}} \leq \omega(x ; F ; V), \quad \forall x \in V .
\end{gathered}
$$

En particulier pour tout $\alpha \in] 0,1[$ et tout compact $K \subset V(F, \alpha)$, il existe $c(K, \alpha)>0$ tel que

$$
\left\|B_{j}\right\|_{K} \leq c(K, \alpha) \mu_{j}^{\alpha}, \quad \forall j \geq 1 .
$$


La première démonstration de la condition (3.1.1) était basée sur le concept délicat de nucléarité (cf. [Mi], [Za2]). Cependant Borchers en a donné indépendamment une démonstration plus simple dans le cas où $F:=\bar{V}_{0}$ est l'adhérence d'un domaine $V_{0} \Subset V$ assez régulier, en considérant une famille à un paramètre complexe de noyaux reproduisants, interpolant convenablement les noyaux de Bergman des deux domaines $V_{0}$ et $V$.

Reprenant une idée analogue dont les prémisses étaient déja dans [Ber], Nguyen Than Van a donné une démonstration plus simple de la condition (3.1.1) lorsque $F:=\bar{V}_{0}$ est l'adhérence d'un domaine $V_{0} \Subset V$ assez régulier et en a déduit cette condition dans le cas général par un argument basé sur le concept de $n$-diamètre dû à Kolmogoroff (cf. [Ng], [Mi]).

Récemment le second auteur a donné une démonstration élémentaire et naturelle du lemme dans un cas assez général suivant les mêmes lignes que [Bo], [Ng], [Ng-Ze2], en déduisant la propriété (3.1.1) de résultats classiques de la théorie spectrale des opérateurs compacts hermitiens définis positifs, dont la construction de Bergman est en fait un cas particulier (cf. [Ze3]). Indiquons simplement les étapes essentielles de cette preuve.

Démonstration du lemme 3.1.1. Soit $J$ l'opérateur de restriction qui à tout $h \in H_{1}$ fait correspondre $h_{\mid F} \in H_{0}$. L'opérateur $J$ est injectif et le théorème de Montel implique que $J$ est compact. Soit $\mathcal{O}(V)$ l'espace des fonctions holomorphes sur $V$ avec la topologie usuelle. Comme $V$ est fortement hyperconvexe, il existe un domaine pseudoconvexe $G \ni V$ et une fonction $\varphi: G \rightarrow]-\infty, 0[$ plurisousharmonique continue telle que $V=\{y \in G: \varphi(y)<0\}$. Le domaine $V$ est alors de Runge dans $G$, i.e. $\overline{\mathcal{O}(G)}=\mathcal{O}(V)$ et comme $\mathcal{O}(G) \subset H_{1} \subset \mathcal{O}(V)$, on en déduit facilement que les injections

$$
\mathcal{O}(G) \hookrightarrow H_{1} \hookrightarrow \mathcal{O}(V) \hookrightarrow H_{0}
$$

sont continues et à images denses.

La construction de la base orthogonale s'obtient alors en appliquant la méthode des systèmes doublement orthogonaux de S. Bergman [Ber] développée dans ce contexte dans [Ng-Ze3]. La condition (3.1.1) sur la suite $\left(\mu_{j}\right)$ se démontre facilement lorsque $F$ est l'adhérence d'un domaine assez régulier contenant $F$ et relativement compact dans $V$, en considérant une famille à un paramètre complexe interpolant les noyaux de Bergman des deux domaines $V_{0}$ et $V_{1}:=V$. Elle s'en déduit facilement dans le cas général en comparant la suite $\mu_{j}(F, V)$ à la suite $\mu_{j}\left(V_{0}, V\right)$ associée à un couple $\left(V_{0}, V\right)$, où $V_{0}$ est un domaine assez régulier contenant $F$ et relativement compact dans $V$ (cf. [Ze3]).

Pour prouver l'estimation (3.1.2), on procède de la façon suivante. D'après la continuité des injections (3.1.4), on montre que la fonction définie par $u(x):=\left(\lim \sup _{j}\left(\log \left|B_{j}\right| / \log \mu_{j}\right)\right)^{*}$ est plurisousharmonique sur $V$ et 
vérifie l'inégalité $u \leq 1$ sur $V$. Par un raisonnement direct et simple basé sur la condition de convergence (3.1.1) (cf. [Ze3]), on montre que $u \leq 0$ $\mu$-presque partout sur $F$, ce qui implique l'inégalité (3.1.2) grâce à la propriété de conservation de la $P$-mesure (2.1.6). Quant aux estimations (3.1.3), elles résultent immédiatement de (3.1.2) en appliquant le lemme de Hartogs (cf. $[\mathrm{Ze} 2])$.

3.2. Fonctions séparément holomorphes. Le résultat suivant constitue l'étape essentielle dans les démonstrations des théorèmes sur l'extension des fonctions séparément holomorphes qui seront énoncés au paragraphe suivant.

Proposition 3.2.1. Soient $U \Subset X, V \Subset Y$ des domaines et $E \subset U$, $F \subset V$ non-pluripolaires. On suppose de plus que $F$ est compact et que $V$ est fortement hyperconvexe. Alors si $f: W \rightarrow \mathbb{C}$ est séparément holomorphe, il existe une fonction $g$ holomorphe sur $\widetilde{W}$ telle que $g=f$ sur $\widetilde{W} \cap W$.

Démonstration. Cela se fait en trois étapes.

1) On suppose $f$ continue et bornée sur $U \times F$. On pose alors

$$
M:=\sup _{x \in U, y \in F}|f(x, y)|<+\infty .
$$

Soient $\mu$ la mesure de Monge-Ampère associée au couple $(F, V), H_{1}:=$ $L^{2}(V, d \lambda) \cap \mathcal{O}(V)$ l'espace de Bergman des fonctions holomorphes dont le module est de carré intégrable sur $V$, et $H_{0}$ la fermeture de $H_{1}$ dans $L^{2}(F, d \mu)$. D'après le lemme 3.1.1, il existe une base doublement orthogonale $\left(B_{j}\right)_{j \geq 1}$ dans $H_{1}$ et $H_{0}$ telle que

$$
\left\|B_{j}\right\|_{H_{0}}=1, \quad\left\|B_{j}\right\|_{H_{1}}=\mu_{j} \nearrow+\infty .
$$

De plus $\sum_{j=1}^{+\infty} \mu_{j}^{-\varepsilon}<+\infty$ pour tout $\varepsilon>0$.

Pour chaque $x \in E$, la fonction $f_{x}=f(x, \cdot)$ est holomorphe sur $V$, il existe donc des coefficients $c_{j}(x)$ tels que

$$
f_{x}(y)=f(x, y)=\sum_{j=1}^{+\infty} c_{j}(x) B_{j}(y) \quad \text { pour tout } y \in V
$$

avec convergence uniforme sur tout compact de $V$. Les coefficients $c_{j}$ sont donnés par la formule

$$
c_{j}(x)=\int_{F} f(x, y) \bar{B}_{j}(y) d \mu(y) .
$$

D'autre part, la fonction

$$
U \ni x \mapsto \int_{F} f(x, y) \bar{B}_{j}(y) d \mu(y)
$$

est holomorphe sur $U$, car $f$ est continue et bornée sur $U \times F$ et $f^{y}=f(\cdot, y)$ est holomorphe sur $U$ pour tout $y \in F$. Notons encore $c_{j}$ cette fonction. 
Alors on a les estimations suivantes :

$$
\left|c_{j}(x)\right| \leq M[\mu(F)]^{1 / 2}, \quad \forall x \in U, \forall j \geq 1,
$$

et

$$
\left|c_{j}(x)\right|=\frac{1}{\mu_{j}^{2}}\left|\int_{V} f(x, y) \bar{B}_{j}(y) d \lambda(y)\right| \leq \frac{\gamma(x)}{\mu_{j}}, \quad \forall x \in E, \forall j \geq 1
$$

où

$$
\gamma(x)=\left(\int_{V}|f(x, y)|^{2} d \lambda(y)\right)^{1 / 2} \quad \text { pour } x \in E .
$$

Il en résulte que la suite

$$
v_{j}:=\frac{\log \left|c_{j}\right|}{\log \mu_{j}}, \quad j \geq 1,
$$

est uniformément majorée sur $U$.

Posons $v:=\lim \sup _{j \rightarrow+\infty} v_{j}$. Les inégalités précédentes entraînent que $v \leq 0$ sur $U$ et $v \leq-1$ sur $E$. Soit $A:=\left\{x \in U: v(x)<v^{*}(x)\right\}$. On a $v^{*}+1 \leq 0$ sur $E \backslash A$ et $v^{*}+1 \leq 1$ sur $U$. Comme $v^{*}$ est faiblement plurisousharmonique sur $U$, il en résulte que $v^{*} \leq \omega^{*}(\cdot ; E \backslash A, U)-1$ sur $U$. Or $A$ est pluripolaire et $U \Subset X$ relativement compact, donc $\omega^{*}(\cdot ; E \backslash A ; U)=$ $\omega^{*}(\cdot ; E ; U)$. Le lemme de Hartogs implique que pour tout $\left.\alpha \in\right] 0,1[$ et tout compact $K \subset U(E, \alpha)$, il existe une constante $c(K, \alpha)$ telle que

$$
\left\|c_{j}\right\|_{K} \leq c(K, \alpha) \mu_{j}^{\alpha-1} \quad \text { pour tout } j \geq 1 .
$$

Soit $\alpha \in] 0,1\left[\right.$ fixé; montrons que la série $\sum_{j=1}^{+\infty} c_{j} B_{j}$ converge normalement sur tout compact de $U(E, \alpha) \times V(F, 1-\alpha)$.

Soient $K \subset U(E, \alpha), L \subset V(F, 1-\alpha)$ des compacts. Il existe $\varepsilon>0$ tel que $K \Subset U(E, \alpha-\varepsilon)$ et $L \Subset V(F, 1-\alpha-\varepsilon)$. On a donc

$$
\left\|c_{j}\right\|_{K}\left\|B_{j}\right\|_{L} \leq c(K, \alpha-\varepsilon) c(L, 1-\alpha-\varepsilon) \mu_{j}^{-2 \varepsilon}, \quad \forall j \geq 1 .
$$

Comme $\sum_{j \geq 0} \mu_{j}^{-2 \varepsilon}<+\infty$, il en résulte que la série $\sum_{j=1}^{+\infty} c_{j} B_{j}$ converge normalement sur tout compact de $U(E, \alpha) \times V(F, 1-\alpha)$. Comme

$$
\bigcup_{0<\alpha<1} U(E, \alpha) \times V(F, 1-\alpha)=\widetilde{W}
$$

on en déduit que cette série converge normalement sur tout compact de $\widetilde{W}$ vers une fonction $g$ holomorphe sur $\widetilde{W}$. Il est clair par définition même que $g=f$ sur $\widetilde{W} \cap W$.

2) On suppose maintenant $E \Subset U$. Soit $\left(U_{s}\right)_{s \geq 1}$ une suite croissante de domaines tels que $E \Subset U_{s} \Subset U$ pour tout $s \geq 1$ et $\bigcup_{s \geq 1} U_{s}=U$. Fixons $s \in \mathbb{N}^{*}$ et pour tout $k \geq 1$, posons

$$
F_{k}=F_{s, k}=\left\{y \in F:|f(x, y)| \leq k, \forall x \in U_{s}\right\} .
$$


Il est clair que $F_{k} \subset F_{k+1}$ et $\bigcup_{k \geq 1} F_{k}=F$, donc il existe $k_{0} \geq 1$ tel que $F_{k}$ soit non-pluripolaire pour tout $k \geq k_{0}$.

On démontre facilement par un argument de famille normale que l'ensemble $F_{k}$ est fermé et que $f$ est continue sur $U_{s} \times F_{k}$.

D'après la première étape, pour tout $k \geq k_{0}$, il existe une fonction holomorphe $g_{k}=g_{k}^{s}$ sur $\widetilde{W}_{k}$ et égale à $f$ sur $\widetilde{W}_{k} \cap W_{k}$, où

$$
W_{k}=W_{k}^{s}:=(E \times V) \cup\left(U_{s} \times F_{k}\right) .
$$

On obtient alors une suite de couples $\left(g_{k}^{s}, \widetilde{W}_{k}^{s}\right)_{k}$ avec $\widetilde{W}_{k}^{s} \subset \widetilde{W}_{k+1}^{s}, \bigcup_{k \geq k_{0}} \widetilde{W}_{k}^{s}$ $=\widetilde{W^{s}}$ et $g_{k}^{s}=f$ sur $\widetilde{W}_{k}^{s} \cap W_{k}^{s}$ pour tout $k \geq k_{0}$.

Par les mêmes arguments que dans la première étape, on montre que $g_{k}^{s}=g_{k+1}^{s}$ sur $\widetilde{W}_{k}^{s} \cap \widetilde{W}_{k+1}^{s}=\widetilde{W}_{k}^{s}$. On peut donc définir une fonction $g^{s}$ sur $\widetilde{W}^{s}=\bigcup_{k>k_{0}} \widetilde{W}_{k}^{s}$ en posant $g^{s}:=g_{k}^{s}$ sur $\widetilde{W}_{k}^{s}$ pour $k \geq 1$. La fonction $g^{s}$ ainsi définie est holomorphe sur $\widetilde{W}^{s}$ et vérifie $g^{s}=f$ sur $\widetilde{W}^{s} \cap W^{s}$. De la même façon, on voit que les éléments $\left(g^{s}, W^{s}\right)$ se recollent en une fonction holomorphe $g$ sur $\widetilde{W}=\bigcup_{s} \widetilde{W}^{s}$ telle que $g=f$ sur $\widetilde{W} \cap W$.

3) Passons au cas général. On écrit alors $E=\bigcup_{k>1} E_{k}$, où $\left(E_{k}\right)_{k}$ est une suite croissante d'ensembles non-pluripolaires et relativement compacts dans $U$. L'étape 2 entraîne l'existence d'une suite de couples $\left(g_{k}, \widetilde{W}_{k}\right)_{k}$, où $g_{k} \in \mathcal{O}\left(\widetilde{W}_{k}\right)$ et telle que $g_{k}=f$ sur $\widetilde{W}_{k} \cap W_{k}$ pour tout $k \geq 1$. En recollant les diverses extensions comme précédemment, on obtient une fonction $\tilde{f} \in$ $\mathcal{O}(\widetilde{W})$ telle que $\widetilde{f}=f$ sur $\widetilde{W} \cap W$.

À partir du résultat précédent on peut démontrer le théorème suivant qui est une généralisation au cas des espaces analytiques singuliers du résultat principal de [Ng-Ze2].

Proposition 3.2.2. Soient $U \Subset X, V \Subset Y$ des domaines relativement compacts et $E \subset U, F \subset V$ des ensembles non-pluripolaires. On suppose que $F$ est un borelien et que $V$ est pseudoconvexe. Alors si $f: W \rightarrow \mathbb{C}$ est séparément holomorphe sur $W$, il existe une fonction $\widetilde{f}$ holomorphe sur $\widetilde{W}$ telle que $\widetilde{f}=f$ sur $\widetilde{W} \cap W$.

Démonstration. On suppose d'abord que $F$ est de type $\mathcal{F}_{\sigma}$.

On peut alors écrire $F=\bigcup_{k>1} F_{k}$ où $\left(F_{k}\right)_{k}$ est une suite croissante de compacts non-pluripolaires. En posant $W_{k}:=(E \times V) \cup\left(U \times F_{k}\right)$ et en appliquant la proposition 3.2.1, on obtient une fonction $g_{k}$ holomorphe sur $\widetilde{W}_{k}$ telle que $g_{k}=f$ sur $\widetilde{W}_{k} \cap W_{k}$. Comme $\bigcup_{k} \widetilde{W}_{k}=\widetilde{W}$, on en déduit comme dans la démonstration de la proposition 3.2.1 que les $g_{k}$ se recollent en une fonction $g$ holomorphe sur $\widetilde{W}$ telle que $g=f$ sur $\widetilde{W} \cap W$. 
Supposons maintenant que $F$ soit un borelien quelconque. Soit $\left(V_{s}\right)_{s}$ une suite croissante de domaines pseudoconvexes relativement compacts dans $V$ telle que $F_{s}:=F \cap V_{s}$ soit non-pluripolaire pour tout $s \geq 1$ et $\bigcup_{s \geq 1} V_{s}=V$.

Soit $s \in \mathbb{N}^{*}$ fixé et soit $t \geq s$. Comme $F$ est un borelien, d'après Bedford et Taylor [Be-Ta], il existe $F_{s}^{\prime} \subset F_{s}$ de type $\mathcal{F}_{\sigma}$ tel que $\omega\left(\cdot ; F_{s}^{\prime} ; V_{t}\right)=$ $\omega\left(\cdot ; F_{s} ; V_{t}\right)$ sur $V_{t}$. On applique la quatrième étape avec $F_{s}^{\prime}$ et $V_{t}$ et on recolle les morceaux pour $t \geq s$. On obtient alors une suite $\left(g_{s}, \widetilde{W}_{s}\right)_{s}$ vérifiant $g_{s}=f$ sur $\widetilde{W}_{s} \cap W_{s}$. Ensuite, en recollant les morceaux pour tous les $s \geq 1$, on obtient un prolongement $\widetilde{f} \in \mathcal{O}(\widetilde{W})$ de $f$ tel que $\widetilde{f}=f$ sur $\widetilde{W} \cap W$.

3.3. Propagation de l'holomorphie. Le but de cette section est de montrer que l'holomorphie se propage à partir d'un petit rectangle ouvert dans la direction où il y a holomorphie partielle. L'étape cruciale est la suivante.

Lemme 3.3.1. Soient $U \subset X$ et $V_{0} \subset V \Subset Y$ des domaines, et $E \subset U$ un ensemble non-pluripolaire. Soit $f: U \times V_{0} \rightarrow \mathbb{C}$ une fonction holomorphe telle que pour tout $x \in E$, la fonction partielle $f(x, \cdot)$ se prolonge holomorphiquement à $V$. Alors pour tout $a \in E^{*}$ et pour tout domaine $G \Subset V$ vérifiant $G \cap V_{0} \neq \emptyset$, il existe un voisinage ouvert connexe $U_{a}$ de a et une fonction $h_{a}$ holomorphe sur $U_{a} \times G$ tels que $h_{a}=f$ sur $U_{a} \times\left(G \cap V_{0}\right)$. En particulier, $f$ s'étend holomorphiquement au voisinage de chaque point de $E^{*} \times V$.

Démonstration. Soit $\mathfrak{D}$ une base dénombrable d'ouverts de $V$ formée de domaines fortement hyperconvexes. Il existe alors une suite finie de domaines $D_{1}, \ldots, D_{r} \in \mathfrak{D}$ tels que $\bar{G} \subset \bigcup_{p=1}^{r} D_{p}$. On peut également choisir des domaines fortement hyperconvexes $\left\{G_{j}: 1 \leq j \leq r\right\}$ tels que $D_{j} \Subset G_{j} \Subset V$ pour $j=1, \ldots, r$. Grâce à la connexité de $G$, quitte à permuter les ouverts $D_{p}$, on peut supposer que $V_{0} \cap D_{1} \neq \emptyset$ et $D_{p} \cap \bigcup_{l=1}^{p-1} D_{l} \neq \emptyset$ pour $2 \leq p \leq r$. Posons $V_{p}=V_{0} \cup D_{1} \cup \ldots \cup D_{p}$ pour $1 \leq p \leq r$ de sorte que les ouverts $V_{p}$ sont tous connexes.

On va construire, par récurrence sur $p(0 \leq p \leq r)$, des ouverts connexes $U_{p}$ contenant $a$ et des fonctions $f_{p} \in \mathcal{O}\left(U_{p} \times V_{p}\right)$ tels que $f_{p}=f$ sur $U_{p} \times V_{0}$. Pour $p=0$, on prend $U_{0}=U$ et $f_{0}=f$. Soit $1 \leq p \leq r$ et supposons construits l'ouvert $U_{p-1}$ et la fonction $f_{p-1} \in \mathcal{O}\left(U_{p-1} \times V_{p-1}\right)$ tels que $f_{p-1}=$ $f$ sur $U_{p-1} \times V_{0}$.

Observons que pour tout $x \in E \cap U_{p-1}$, l'application partielle $f_{x}$ se prolonge en une fonction holomorphe $\tilde{f}_{x}$ sur $V$ et qu'alors $\widetilde{f}_{x}(y)=f_{p-1}(x, y)$ pour tout $(x, y) \in E \cap U_{p-1} \subset V_{p-1}$. Posons $E_{p-1}:=E \cap U_{p-1} \subset U_{p-1}$ et $F_{p}:=D_{p} \cap V_{p-1} \subset G_{p}$. On peut donc définir une fonction $h$ sur l'ensemble

$$
W_{p}:=\left(U_{p-1} \times F_{p}\right) \cup\left(E_{p-1} \times G_{p}\right)
$$


en posant $h(x, y):=\widetilde{f}_{x}(y)$ si $(x, y) \in E_{p-1} \times G_{p}$ et $h(x, y):=f_{p-1}(x, y)$ si $(x, y) \in U_{p-1} \times F_{p}$.

Comme $U_{p-1}$ est pseudoconvexe, il résulte de la proposition 3.2.2 qu'il existe une fonction $\widetilde{h}$ holomorphe sur l'ouvert

$$
\widetilde{W}_{p}:=\left\{(x, y) \in U_{p-1} \times G_{p}: \omega\left(x ; E_{p-1} ; U_{p-1}\right)+\omega\left(y ; F_{p} ; G_{p}\right)<1\right\}
$$

telle que $\widetilde{h}=h$ sur $\widetilde{W}_{p} \cap W_{p}$. Remarquons que $U_{p-1} \times F_{p} \subset \widetilde{W}_{p} \cap W_{p}$ et que donc $\widetilde{h}=h=f_{p-1}$ sur $U_{p-1} \times F_{p}$. Par conséquent on a $\widetilde{h}=f_{p-1}$ sur $U_{p-1} \times\left(D_{p} \cap V_{p-1}\right)$. Comme $D_{p} \Subset G_{p}$, il en résulte qu'il existe un domaine pseudoconvexe $U_{p} \Subset U_{p-1}$ voisinage de $a$ tel que $U_{p} \times D_{p} \subset \widetilde{W}_{p}$. Il en résulte que l'on peut donc définir une fonction $f_{p}$ sur $U_{p} \times V_{p}$ en posant

$$
f_{p}= \begin{cases}\widetilde{h} & \text { sur } U_{p} \times D_{p}, \\ f_{p-1} & \text { sur } U_{p} \times V_{p-1} .\end{cases}
$$

Il est clair que $f_{p}$ est holomorphe sur $U_{p} \times V_{p}$ et que $f_{p}=f$ sur $U_{p} \times V_{0}$. Ainsi pour $p=r$ on obtient une fonction $f_{r}$ holomorphe sur $U_{r} \times V_{r}$ telle que $f_{r}=f$ sur $U_{r} \times V_{0}$. En posant $U_{a}:=U_{r}$, la restriction $h_{a}$ de $f_{r}$ à $U_{a} \times G$ est la fonction cherchée.

En appliquant les résultats précédents, on va montrer que si $f$ est une fonction séparément holomorphe sur $W$ alors elle admet une extension holomorphe au voisinage de chaque point de $W^{*}$, sans hypothèse sur les facteurs. Plus précisément, on a le résultat suivant.

ThÉORÈme 3.3.2. Soient $U \subset X, V \subset Y$ des domaines, $E \subset U, F \subset V$ des ensembles non-pluripolaires et soit $f: W \rightarrow \mathbb{C}$ une fonction séparément holomorphe sur $W$. Alors $f$ se prolonge holomorphiquement au voisinage de chaque point de $W^{*}:=\left(U \times F^{*}\right) \cup\left(E^{*} \times V\right)$.

Démonstration. En effet, soient $a \in E^{*}, U_{1} \Subset U$ un domaine quelconque contenant $a$ et $V_{1} \Subset V$ un domaine hyperconvexe tel que $F \cap V_{1}$ soit non-pluripolaire. Posons $E_{1}:=E \cap U_{1}$ et définissons, pour chaque $k \geq 1$, l'ensemble $F_{k}:=\left\{y \in F: \sup _{x \in U_{1}}|f(x, y)| \leq k\right\}$. Alors $F=\bigcup_{j} F_{j}$ et comme $F$ est non-pluripolaire, il existe $k_{0} \geq 1$ tel que $F_{k}$ soit non-pluripolaire pour tout $k \geq k_{0}$.

Posons $F_{0}:=F_{k_{0}}$ et soit $V_{2} \Subset V_{1}$ un domaine fortement hyperconvexe tel que $F_{1}:=F_{0} \cap V_{2}$ soit non-pluripolaire. L'ensemble $L_{1}:=\bar{F}_{1}=\overline{F_{0} \cap V_{2}} \subset V_{1}$ est alors un compact non-pluripolaire dans $V_{1}$.

Soient $y_{0} \in L_{1}$ et $\left(y_{k}\right)_{k}$ une suite de points de $F_{1}$, convergeant vers $y_{0}$. Considérons la suite de fonctions définie par $g_{k}(x):=f\left(x, y_{k}\right)$ pour $k \geq 1$ et $x \in U_{1}$. Pour tout $k \geq 1, g_{k}$ est holomorphe sur $U_{1}$ et $\left|g_{k}\right| \leq c$ dans $U_{1}$. D'après le théorème de Montel, quitte à passer à une sous-suite, on peut supposer que la suite $\left(g_{k}\right)$ converge vers une fonction $g^{y_{0}}$ holomorphe sur $U_{1}$. Il est clair que $g^{y_{0}}=f\left(\cdot, y_{0}\right)$ sur $E_{1}$. De plus la fonction $g^{y_{0}}$ ne dépend 
que du point $y_{0}$ mais non de la suite $\left(y_{k}\right)_{k}$ de $F_{1}$ qui converge vers $y_{0}$. En effet, soit $\left(w_{j}\right)_{j} \subset F_{1}$ une autre suite convergeant vers $y_{0}$ et telle que la suite de fonctions holomorphes correspondante converge vers une fonction holomorphe $h^{y_{0}}$ sur $U_{1}$. Alors on aura $g^{y_{0}}=f\left(\cdot, y_{0}\right)=h^{y_{0}}$ sur $E_{1}$. D'après le principe du prolongement analytique généralisé on a $g^{y_{0}}=h^{y_{0}}$ sur $U_{1}$, puisque $E_{1}$ est non-pluripolaire et que $U_{1}$ est un ouvert connexe.

Soient $W_{1}:=\left(U_{1} \times L_{1}\right) \cup\left(E_{1} \times V_{1}\right)$ et $g: W_{1} \rightarrow \mathbb{C}$ la fonction définie par

$$
g(x, y)= \begin{cases}g^{y}(x) & \text { si }(x, y) \in U_{1} \times L_{1}, \\ f(x, y) & \text { si }(x, y) \in E_{1} \times V_{1} .\end{cases}
$$

Alors $g$ est séparément holomorphe sur $W_{1}$.

Ainsi $L_{1} \subset V_{1}$ est un compact non-pluripolaire, $V_{1}$ est un domaine fortement hyperconvexe et $g$ est une fonction séparément holomorphe sur $W_{1}$. D'après la proposition 3.2.1, pour tout $\left.\alpha \in\right] 0,1[$, il existe une fonction $g_{\alpha}$ holomorphe sur $U_{1}\left(E_{1}, \alpha\right) \times V_{1}\left(L_{1}, 1-\alpha\right)$ telle que $g_{\alpha}=g$ sur $W_{1} \cap\left(U_{1}\left(E_{1}, \alpha\right) \times V_{1}\left(L_{1}, 1-\alpha\right)\right)$.

Ainsi la fonction $g_{\alpha}$ est holomorphe sur le rectangle $U_{1}\left(E_{1}, \alpha\right) \times$ $V_{1}\left(L_{1}, 1-\alpha\right)$ et pour tout $x \in E_{1}^{*} \subset U_{1}\left(E_{1}, \alpha\right)$, la fonction partielle $g_{\alpha}(x, \cdot)=$ $g(x, \cdot)=f(x, \cdot)$ sur $V_{1}\left(L_{1}, 1-\alpha\right)$ se prolonge en une fonction holomorphe sur $V$. Le lemme 3.3.1 entraîne que $g_{\alpha}$, et donc $f$, a une extension holomorphe au voisinage ouvert de chaque point de $E_{1}^{* *} \times V=\left(E^{*} \cap U_{1}\right) \times V$. Comme $U_{1}$ est un voisinage d'un point arbitraire de $E^{*}$, il en résulte que $f$ s'étend holomorphiquement au voisinage de chaque point de $E^{*} \times V$. Par symétrie, on en déduit que $f$ s'étend holomorphiquement au voisinage de chaque point de $W^{*}$.

3.4. Extension des fonctions séparément holomorphes. Le but de ce paragraphe est de démontrer deux résultats importants sur l'extension des fonctions séparément holomorphes.

On dira qu'un domaine $U \subset X$ possède une extension holomorphe pseudoconvexe s'il existe un domaine pseudoconvexe $\widetilde{U}$ d'un espace analytique $\widetilde{X}$ tel que toute fonction holomorphe sur $U$ se prolonge en une fonction holomorphe sur $\widetilde{U}$.

THÉORÈme 3.4.1. Soient $X, Y$ des espaces analytiques complexes, $U \subset X$, $V \subset Y$ des domaines et $E \subset U, F \subset V$ des ensembles non-pluripolaires et $W:=W(E \times F ; U \times V)$. Soit $f: W \rightarrow \mathbb{C}$ une fonction séparément holomorphe sur $W$. Alors il existe un voisinage ouvert $\Omega$ de $W^{*}$ dans $U \times V$ et une fonction $\widetilde{f}$ holomorphe sur $\Omega$ telle que $\tilde{f}=f$ sur $\Omega \cap W$.

THÉORÈme 3.4.2. Soient $X, Y$ des espaces analytiques complexes, $U \subset X$, $V \subset Y$ des domaines, $E \subset U, F \subset V$ des ensembles non-pluripolaires et $W:=(U \times F) \cup(E \times V)$. Alors on a les propriétés suivantes : 
1) Si $U$ ou $V$ possède une extension holomorphe pseudoconvexe, alors pour toute fonction $f: W \rightarrow \mathbb{C}$ séparément analytique sur $W$, il existe une fonction $\widetilde{f}$ holomorphe sur $\widetilde{W}$ telle que $\widetilde{f}=f$ sur $\widetilde{W} \cap W$.

2) Si de plus $X$ et $Y$ sont des variétés de Stein, alors l'enveloppe d'holomorphie de $W$ coïncide avec la variété de Stein suivante:

$$
\widehat{W}:=\{(x, y) \in \widehat{U} \times \widehat{V}: \widetilde{\omega}(x ; E ; \widehat{U})+\widetilde{\omega}(y ; F ; \widehat{V})<1\}
$$

où $\widehat{U}$ et $\widehat{V}$ sont les enveloppes d'holomorphie de $U$ et $V$ respectivement.

Le théorème 3.4.1 sera une conséquence immédiate du théorème 3.3.2 et du résultat suivant que nous avons préféré énoncer sous une forme générale de façon à l'utiliser de nouveau au paragraphe 4 .

Proposition 3.4.3. Soient $X, Y, Z$ des espaces analytiques complexes, $U \subset X, V \subset Y$ des domaines, $A \subset U, B \subset V$ des ensembles nonpluripolaires. Soit $f: \Sigma:=(U \times B) \cup(A \times V) \rightarrow Z$ une application qui s'étend holomorphiquement au voisinage de chaque point de $\Sigma$. Alors, si on pose $A^{\prime}:=A \cap A^{*}$ et $B^{\prime}:=B \cap B^{*}$, il existe un voisinage ouvert $\Omega$ de $\Sigma^{\prime}:=\left(U \times B^{\prime}\right) \cup\left(A^{\prime} \times V\right)$ et une application holomorphe $\widetilde{f}$ de $\Omega$ dans $Z$ telle que $\tilde{f}=f$ sur $\Omega \cap \Sigma$.

Démonstration. La démonstration de ce résultat se fait en plusieurs étapes.

ÉTAPE 1. On démontre tout d'abord que pour tout domaine $G \Subset V$ tel que $G \cap B^{*} \neq \emptyset$, il existe un voisinage consistant $U^{\prime} \subset U$ de $A^{\prime}$ et une application holomorphe $h$ sur $U^{\prime} \times G$ telle que $h=f$ sur $\left(U^{\prime} \times G\right) \cap \Sigma$. En effet, fixons un point $a \in A^{\prime}=A \cap A^{*}$. D'après l'hypothèse, pour tout $b \in \bar{G}$, il existe un domaine $U^{b} \subset U$ voisinage de $a$, un domaine $V^{b} \subset V$ tel que $V^{b} \cap B^{*} \neq \emptyset$ voisinage de $b$ et une application $h^{b}$ holomorphe sur $U^{b} \times V^{b}$ telle que $h^{b}=f$ sur $\left(U^{b} \times V^{b}\right) \cap \Sigma$. Par compacité, on peut recouvrir $\bar{G}$ par un nombre fini de domaines $V^{b_{i}}(1 \leq i \leq m)$. En posant $U_{a}:=\bigcap_{i=1}^{m} U^{b_{i}}$, on obtient un voisinage ouvert connexe de $a$ tel que $h^{b_{i}}=f \operatorname{sur}\left(A \cap U_{a}\right) \times V^{b_{i}}$ pour chaque $i=1, \ldots, m$. Ainsi pour $i, k$ compris entre 1 et $m$, on a $h^{b_{i}}=h^{b_{k}}$ $\operatorname{sur}\left(A \cap U_{a}\right) \times\left(V^{b_{i}} \cap V^{b_{k}}\right)$. Soit $V_{a}$ la composante connexe de l'ouvert $\bigcup_{i=1}^{m} V^{b_{i}}$ qui contient $\bar{G}$. Comme $U_{a} \cap A$ est non-pluripolaire et que $U_{a}$ est connexe, il en résulte, grâce au principe du prolongement analytique généralisé, que les applications holomorphes $h^{b_{i}}$ se recollent en une application $h_{a}$ holomorphe sur $U_{a} \times V_{a}$ telle que $h_{a}=f$ sur $\left(A \cap U_{a}\right) \times G$.

Le but maintenant est de recoller les extensions holomorphes $h_{a}$ de $f$ à $U_{a} \times G$ obtenues précédemment. Remarquons que $h_{a}=f$ sur $\left(A \cap U_{a}\right)$ $\times(B \cap G)$ et puisque $A \cap U_{a}$ est non-pluripolaire dans le domaine $U_{a}$, il résulte du théorème de prolongement analytique généralisé que $h_{a}=f$ sur $U_{a} \times(B \cap G)$. Soient maintenant $a, b \in A^{\prime}$ deux points distincts tels que 
$U_{a} \cap U_{b} \neq \emptyset$, où $U_{a} \subset U$ et $U_{b} \subset U$ sont des domaines voisinages de $a$ et $b$ respectivement tels qu'il existe des extensions holomorphes $h_{a}$ et $h_{b}$ de $f$ aux ouverts $U_{a} \times G$ et $U_{b} \times G$ respectivement. Alors $h_{a}=f=h_{b}$ sur $\left(U_{a} \cap U_{b}\right) \times(B \cap G)$. Comme $B \cap G$ est non-pluripolaire et $G$ est connexe, on en déduit que $h_{a}=h_{b}$ sur $\left(U_{a} \cap U_{b}\right) \times G$. En posant $U^{\prime}:=\bigcup_{a \in A^{\prime}} U_{a}$, on obtient alors un voisinage consistant de $A^{\prime}$ et une application holomorphe $h$ sur $U^{\prime} \times G$ telle que $h=f$ sur $\left(U^{\prime} \times G\right) \cap \Sigma$.

ÉtAPe 2. Passons maintenant au cas général. Pour se ramener à l'étape 1 , considérons deux suites exhaustives $\left(U_{j}\right)_{j}$ et $\left(V_{j}\right)_{j}$ de domaines relativement compacts de $U$ et $V$ respectivement. Posons $A_{j}:=A \cap U_{j}, B_{j}:=B \cap V_{j}$ et $\Sigma_{j}:=\left(U_{j} \times B_{j}\right) \cup\left(A_{j} \times V_{j}\right)$ pour $j \geq 1$ et désignons par $f_{j}$ la restriction de $f$ à la croix $\Sigma_{j}$. Il existe alors $j_{0} \geq 1$ tel que $A_{j}$ et $B_{j}$ soit non-pluripolaires dans $X$ et $Y$ respectivement. D'après la première étape, pour chaque $j \geq j_{0}$, il existe des voisinages consistants $U_{j}^{\prime} \subset U_{j}$ et $V_{j}^{\prime} \subset V_{j}$ de $A_{j}^{\prime}:=A_{j} \cap A_{j}^{*}$ et $B_{j}^{\prime}:=B_{j} \cap B_{j}^{*}$ respectivement et une application $h_{j}$ holomorphe sur l'ouvert $\Omega_{j}:=\left(U_{j}^{\prime} \times V_{j}\right) \cup\left(U_{j} \times V_{j}^{\prime}\right)$ à valeurs dans $Z$ telle que $h_{j}=f_{j}$ sur $\Omega_{j} \cap \Sigma_{j}$. Montrons que les applications holomorphes $h_{j}: \Omega_{j} \rightarrow Z$ se recollent en une application holomorphe $h$ sur l'ouvert $\Omega:=\bigcup_{j} \Omega_{j}$. En effet, fixons deux indices $j$ et $k$ tels que $k \geq j \geq j_{0}$, de sorte que l'on a la formule suivante :

$$
\Omega_{k} \cap \Omega_{j}=R_{1} \cup R_{2} \cup R_{3} \cup R_{4},
$$

où

$$
\begin{array}{ll}
R_{1}:=\left(U_{j}^{\prime} \cap U_{k}^{\prime}\right) \times V_{j}, & R_{2}:=U_{j}^{\prime} \times\left(V_{j} \cap V_{k}^{\prime}\right), \\
R_{3}:=\left(U_{j} \cap U_{k}^{\prime}\right) \times V_{j}^{\prime} & R_{4}:=U_{j} \times\left(V_{j}^{\prime} \cap V_{k}^{\prime}\right) .
\end{array}
$$

Remarquons que $A_{j}^{\prime}=A_{j} \cap A_{j}^{*}=A \cap A^{*} \cap U_{j}$ et $B_{j}^{\prime}=B \cap B^{*} \cap V_{j}$ de sorte que $A_{j}^{\prime} \times B_{j}^{\prime}$ est contenu dans chacun des rectangles $R_{i}(1 \leq i \leq 4)$. De plus, pour un rectangle donné $R=R_{i}$ avec $1 \leq i \leq 4$, l'une de ses projections est un voisinage consistant de la projection correspondante de l'ensemble $A_{j}^{\prime} \times B_{j}^{\prime}$ et l'on a $h_{k}=f=h_{j}$ sur $\Omega_{k} \cap \Omega_{j} \cap \Sigma_{j}$. Alors, en appliquant le principe du prolongement analytique généralisé aux restrictions de $h_{j}$ et $h_{k}$ au rectangle $R$ par rapport à l'un des facteurs, on conclut que $h_{k}=h_{j}$ sur $R$.

En effet, considérons le premier rectangle $R_{1}=\left(U_{j}^{\prime} \cap U_{k}^{\prime}\right) \times V_{j}$. Comme $\left(U_{j}^{\prime} \cap U_{k}^{\prime}\right) \times B_{j}^{\prime} \subset \Sigma_{j}$, on a $h_{k}=f=h_{j}$ sur $\left(U_{j}^{\prime} \cap U_{k}^{\prime}\right) \times B_{j}^{\prime} \subset R_{1}$. Comme $B_{j}^{\prime} \subset V_{j}$ est non-pluripolaire et que $V_{j}$ est connexe, il résulte du principe du prolongement analytique généralisé par rapport au second facteur que $h_{k}=h_{j} \operatorname{sur}\left(U_{j}^{\prime} \cap U_{k}^{\prime}\right) \times V_{j}$. Le même raisonnement vaut pour le rectangle $R_{3}$.

Considérons maintenant le rectangle $R_{2}=U_{j}^{\prime} \times\left(V_{j} \cap V_{k}^{\prime}\right)$. Comme $A_{j}^{\prime} \times$ $\left(V_{j} \cap U_{j}^{\prime}\right) \subset \Sigma_{j}$, on a $h_{k}=f=h_{j}$ sur $A_{j}^{\prime} \times\left(V_{j} \cap U_{j}^{\prime}\right) \subset R_{2}$. Comme $U_{j}^{\prime}$ est un voisinage consistant de $A_{j}^{\prime}$, il résulte du principe du prolongement analytique généralisé par rapport au premier facteur que $h_{k}=h_{j}$ sur $U_{j}^{\prime} \times\left(V_{j} \cap V_{k}^{\prime}\right)$. Le même raisonnement vaut pour le rectangle $R_{4}$. 
Par conséquent, les applications holomorphes $h_{j}$ se recollent en une application holomorphe sur $\Omega=\bigcup_{j} \Omega_{j}$ qui étend l'application $f$. De plus, en posant $\Sigma_{j}^{\prime}:=\left(A_{j}^{\prime} \times V_{j}\right) \cup\left(U_{j} \times B_{j}^{\prime}\right), \Omega_{j}$ est un voisinage de $\Sigma_{j}^{\prime}$ et donc $\Omega$ est un voisinage de $\Sigma^{\prime}=\bigcup_{j} \Sigma_{j}^{\prime}$. Ceci achève la démonstration de notre résultat.

Nous pouvons maintenant démontrer facilement le théorème 3.4.1.

Démonstration du théorème 3.4.1. En effet, d'après le théorème 3.3.2, $f$ s'étend holomorphiquement au voisinage de chaque point de $W^{*}=$ $\left(U \times F^{*}\right) \cup\left(E^{*} \times V\right)$. En observant que $A:=E^{*}$ et $B:=F^{*}$ sont des ensembles non-pluripolaires dans $X$ et $Y$ respectivement et en posant $\Sigma:=$ $(U \times B) \cup(A \times V)$, on déduit de la proposition 3.4.3 qu'il existe un voisinage ouvert de $\Sigma^{\prime}:=\left(U \times B^{\prime}\right) \cup\left(A^{\prime} \times V\right)$ auquel la fonction $f$ s'étend holomorphiquement, où $A^{\prime}:=A \cap A^{*}$ et $B^{\prime}:=B \cap B^{*}$. Remarquons maintenant que $A^{\prime}=E^{*}, B^{\prime}=F^{*}$ et donc $\Sigma^{\prime}=W^{*}$, ce qui prouve le résultat voulu.

Passons maintenant à la démonstration du théorème 3.4.2.

Démonstration du théorème 3.4.2. 1) Pour démontrer la première assertion, on procède en trois étapes.

Étape 1. Supposons que $U \Subset X$ et $V \Subset Y$ sont des domaines relativement compacts et que $V$ est pseudoconvexe. Le théorème 3.4.1 implique qu'il existe un voisinage ouvert $\Omega$ de $W^{*}$ et une fonction $g$ holomorphe sur $\Omega$ telle que $g=f$ sur $\Omega \cap W$. Comme la fonction $g$ est séparément holomorphe sur $W^{*}$ et que $E^{*}$ et $F^{*}$ sont des ensembles boreliens non-pluripolaires, la proposition 3.2.2 implique que $g$ a une extension holomorphe $\widetilde{f}$ à $\widetilde{W}$.

ÉtaPe 2. Supposons que $V$ est pseudoconvexe. Soient $\left(U_{j}\right)_{j}$ et $\left(V_{j}\right)_{j}$ deux suites exhaustives de domaines relativement compacts de $U$ et $V$ respectivement telles que les domaines $V_{j}$ soient pseudoconvexes. Posons $E_{j}:=E \cap U_{j}, F_{j}:=F \cap V_{j}$ et $W_{j}:=\left(U_{j} \times F_{j}\right) \cup\left(E_{j} \times V_{j}\right)$ pour $j \geq 1$. Il existe alors $j_{0} \geq 1$ tel que $E_{j}$ et $F_{j}$ soient non-pluripolaires pour tout $j \geq j_{0}$. On voit facilement que $E^{*} \subset \bigcup_{j \geq j_{0}} E_{j}^{*}$ et $F^{*} \subset \bigcup_{j \geq j_{0}} F_{j}^{*}$. D'après la première étape, pour $j \geq j_{0}$, il existe une fonction $\widetilde{f}_{j}$ holomorphe sur $\widetilde{W}_{j}$ telle que $\widetilde{f}_{j}=f$ sur $\widetilde{W}_{j} \cap W_{j}$. Remarquons que $\widetilde{W}_{j} \subset \widetilde{W}_{j+1}$ et que $\widetilde{f}_{j+1}=f=\widetilde{f}_{j}$ sur $\widetilde{W}_{j} \cap W_{j}$. D'après le principe du prolongement analytique généralisé, on en déduit aisément que $\widetilde{f}_{j+1}=\widetilde{f}_{j}$ sur $\widetilde{W}_{j}$, ce qui prouve que les fonctions $\widetilde{f}_{j}$ se recollent en une fonction holomorphe sur $\bigcup_{j} \widetilde{W}_{j}$ qui étend $f$. Posons $u_{j}:=\omega\left(\cdot ; E_{j}^{*} ; U_{j}\right)$ pour $j \geq 1$. La suite $\left(u_{j}\right)_{j}$ décroît vers une fonction plurisousharmonique $u \leq 1$ sur $U$. De plus, il est clair que $u_{j} \leq 0$ sur $E_{j}^{*}$ pour tout $j \geq 1$, ce qui implique que $u \leq 0$ sur $E^{*}$ et par suite $u \leq$ $\omega\left(\cdot ; E^{*} ; U\right)$. De même, si on définit $v_{j}:=\omega\left(\cdot ; F_{j}^{*} ; V_{j}\right)$, on obtient une suite décroissante de fonctions plurisousharmoniques qui converge vers une fonc- 
tion $v$ plurisousharmonique sur $V$ vérifiant $v \leq \omega^{*}\left(\cdot ; F^{*} ; V\right)$. Par conséquent $\widetilde{W} \subset \bigcup_{j} \widetilde{W}_{j}$, ce qui démontre que $f$ a une extension holomorphe à $\widetilde{W}^{*}$.

ÉTAPE 3. Si $\widehat{V}$ est une extension pseudoconvexe de $V$, toute fonction holomorphe sur $V$ se prolonge en une fonction holomorphe sur $\widehat{V}$. Par conséquent la fonction $f$ séparément holomorphe sur $W$ se prolonge en une application séparément holomorphe sur la croix $W^{\prime}:=(U \times F) \cup(E \times \widehat{V})$. D'après l'étape 2 , il en résulte que $f$ s'étend holomorphiquement à l'ouvert $\widetilde{W^{\prime}}$. Comme $\widetilde{W} \subset \widetilde{W^{\prime}}$, le résultat cherché en découle.

2) Si $X$ et $Y$ sont des variétés de Stein, alors les domaines $U$ et $V$ ont des enveloppes d'holomorphie respectives $\widehat{U}$ et $\widehat{V}$ qui sont des variétés de Stein. Il est alors clair que $f$ se prolonge en une application séparément holomorphe sur $W^{\prime}:=\left(\widehat{U} \times F^{*}\right) \cup\left(E^{*} \times \widehat{V}\right)$ qui, d'après la première partie de la démonstration, s'étend holomorphiquement à $\widetilde{W^{\prime}}=\widehat{W}$. Comme $\widehat{W}$ est pseudoconvexe dans la variété de Stein $\widehat{U} \times \widehat{V}$, c'est une variété de Stein, ce qui prouve notre assertion.

4. Applications séparément holomorphes. Nous allons maintenant utiliser les résultats de la section 3 pour démontrer les résultats d'extension pour les applications séparément holomorphes énoncés au paragraphe 2 .

4.1. Propagation de l'holomorphie pour les applications. Soient $X, Y$, $Z$ des espaces analytiques complexes. Le résultat qui suit constitue l'étape essentielle dans la démonstration du théorème de Hartogs pour les applications séparément holomorphes. C'est l'analogue pour les applications du lemme 3.3.1.

Lemme 4.1.1. Soient $K \subset X, L \subset Y$ des ensembles boreliens de type $\mathcal{F}_{\sigma}, U_{0} \subset X$ et $V_{0} \subset Y$ des domaines tels que $K \cap U_{0}$ et $L \cap V_{0}$ soient non-pluripolaires dans $X$ et $Y$ respectivement et $E \subset K \cap U_{0}$ un ensemble non-pluripolaire. Soient $Z$ un espace analytique complexe et $f: U_{0} \times V_{0} \rightarrow Z$ une application holomorphe telle que pour chaque point $x \in E$, l'application partielle $f(x, \cdot): L \cap V_{0} \rightarrow Z$ se prolonge en une application holomorphe d'un voisinage de $L$ dans $Z$. Alors il existe un ensemble borelien $E^{\prime} \subset E^{*}$ quasiégal à $E$ et tel que l'application $f$ s'étend holomorphiquement au voisinage de chaque point de $E^{\prime} \times L$ dans $X \times Y$.

Démonstration. La démonstration se fait en deux étapes.

ÉTAPE 1. Soit $G \subset L$ un compact connexe tel que $V_{0} \cap G$ soit nonpluripolaire. On va montrer qu'il existe un ensemble borelien $E^{\prime} \subset E^{*}$ quasiégal à $E$, un voisinage consistant $U^{\prime} \subset U_{0}$ de $E^{\prime}$, un voisinage ouvert $V \subset Y$ de $G$ et une application holomorphe $h$ de $U^{\prime} \times V$ dans $Z$ tels que $h=f$ sur $U^{\prime} \times\left(V \cap V_{0}\right)$. En effet, considérons une base dénombrable d'ouverts $\mathfrak{D}$ 
de $Y$ et une base dénombrable d'ouverts de $Z$ formée par des domaines de Stein $\left(Z_{l}\right)_{l \geq 1}$ telle que pour chaque $l$, il existe un plongement $\varphi_{l}$ de $Z_{l}$ sur un sous-ensemble analytique du polydisque unité de $\mathbb{C}^{N_{l}}$.

Pour chaque entier $r \in \mathbb{N}^{*}$, on désigne par $\mathcal{H}_{r}$ l'ensemble des applications holomorphes $h$ de $V_{0}$ dans $Z$ pour lesquelles il existe des multi-indices $j:=$ $\left(j_{1}, \ldots, j_{r}\right), k:=\left(k_{1}, \ldots, k_{r}\right) \in\left(\mathbb{N}^{*}\right)^{r}$ tels que les ouverts $D_{j_{1}}, \ldots, D_{j_{r}} \in \mathfrak{D}$ recouvrent $G$ et il existe une application $\widetilde{h}$ holomorphe de $D_{j_{1}} \cup \ldots \cup D_{j_{r}}$ dans $Z$ telle que $\widetilde{h}\left(D_{j_{p}}\right) \subset Z_{k_{p}}$ pour $1 \leq p \leq r$ et $\widetilde{h}=h$ sur $\left(D_{j_{1}} \cup \ldots \cup D_{j_{r}}\right) \cap V_{0}$. On désigne alors par $E_{r}$ l'ensemble des $x \in E$ tels que $f_{x} \in \mathcal{H}_{r}$. Il est facile de vérifier à partir des hypothèses que $\left(E_{r}\right)$ est une suite croissante d'ensembles tels que $E=\bigcup_{r \in \mathbb{N}^{*}} E_{r}$. Comme $E$ est non-pluripolaire, il existe $s \geq 1$ tel que pour tout $r \geq s, E_{r}$ soit non-pluripolaire. Fixons $r \geq s$ et $a \in E_{r}^{*}$. Choisissons des domaines $D_{p}^{\prime}$ tels que $D_{p}^{\prime} \Subset D_{j_{p}}$ pour $1 \leq p \leq r$ et $G \subset D_{1}^{\prime} \cup \ldots \cup D_{r}^{\prime}$. Par connexité de $G$, quitte à permuter les $D_{p}^{\prime}(1 \leq p \leq r)$, on peut supposer que $V_{0} \cap D_{1}^{\prime} \neq \emptyset$ et $D_{p}^{\prime} \cap \bigcup_{1 \leq j \leq p-1} D_{j}^{\prime} \neq \emptyset$ pour $2 \leq p \leq r$. Alors pour chaque $1 \leq p \leq r$, l'ensemble défini par $V_{p}:=V_{0} \cup D_{1}^{\prime} \cup \ldots \cup D_{p}^{\prime}$ est un domaine. On va construire, par récurrence sur $p(0 \leq p \leq r)$, des voisinages ouverts $U_{p} \subset U_{0}$ de $a$ tels que $f$ se prolonge en une application holomorphe de $U_{p} \times V_{p}$ dans $Z$.

Pour $p=0$, la fonction $f_{0}:=f$ est holomorphe sur $U_{0} \times V_{0}$.

Supposons que pour un entier $p(1 \leq p \leq r)$ on a construit un voisinage ouvert $U_{p-1} \subset U_{0}$ de $a$ et une application holomorphe $\widetilde{f}$ de $U_{p-1} \times V_{p-1}$ dans $Z$ telle que $\tilde{f}=f$ sur $\left(E \cap U_{p-1}\right) \times V_{0}$. En choisissant un domaine $D_{p}^{\prime \prime} \Subset D_{p}^{\prime} \cap V_{p-1}$, il résulte alors de la définition de $E_{r}$ que $\widetilde{f}\left(\left(E_{r} \cap U_{p-1}\right) \times D_{p}^{\prime \prime}\right)$ $\subset Z_{k_{p}}$. Par suite, la continuité de $\tilde{f}$ sur $U_{p-1} \times V_{p-1}$ et la compacité de $\overline{D_{p}^{\prime \prime}}$ impliquent qu'il existe un voisinage ouvert connexe $U_{p}^{\prime} \subset U_{p-1}$ de $a$ tel que $\tilde{f}\left(U_{p}^{\prime} \times D_{p}^{\prime \prime}\right) \subset Z_{k_{p}}$. Considérons maintenant l'application holomorphe $g:=\varphi_{p} \circ \tilde{f}: U_{p}^{\prime} \times D_{p}^{\prime \prime} \rightarrow \mathbb{C}^{N_{p}}$, où $\varphi_{p}$ est un plongement de $Z_{k_{p}}$ sur un sous-ensemble analytique du polydisque unité de $\mathbb{C}^{N_{p}}$. Par définition de $E_{r}$, pour tout $x \in E_{r} \cap U_{p}^{\prime}=E_{r} \cap U_{p-1}^{\prime}$ l'application partielle $g(x, \cdot)$ se prolonge en une application holomorphe de $D_{j_{p}}$ dans $\mathbb{C}^{N_{p}}$. Comme $D_{p}^{\prime} \Subset D_{j_{p}}$, il résulte du lemme 3.3.1 qu'il existe un domaine $U_{p} \subset U_{p}^{\prime}$ voisinage de $a$ et une application holomorphe $\widetilde{g}: U_{p} \times D_{p}^{\prime} \rightarrow \mathbb{C}^{N_{p}}$ telle que $\widetilde{g}=g$ sur $U_{p} \times D_{p}^{\prime \prime}$. Comme $\varphi_{p}\left(Z_{k_{p}}\right)$ est un sous-ensemble analytique fermé du polydisque unité de $\mathbb{C}^{N_{p}}$ et que $\widetilde{g}\left(\left(E_{r} \cap U_{p}\right) \times D_{p}^{\prime \prime}\right)=g\left(\left(E_{r} \cap U_{p}\right) \times D_{p}\right) \subset \varphi_{p}\left(Z_{k_{p}}\right)$, alors, puisque $E_{r} \cap U_{p}$ est non-pluripolaire dans le domaine $U_{p}$, le principe du prolongement analytique généralisé entraîne que $\widetilde{g}\left(U_{p} \times D_{p}^{\prime}\right) \subset \varphi_{p}\left(Z_{k_{p}}\right)$.

Posons $f^{\prime}:=\varphi_{p}^{-1} \circ \widetilde{g}: U_{p} \times D_{p}^{\prime} \rightarrow Z_{k_{p}} \subset Z$. Alors d'une part $f^{\prime}$ est holomorphe sur $U_{p} \times D_{p}^{\prime}$ et $f^{\prime}=\widetilde{f} \operatorname{sur} U_{p} \times D_{p}^{\prime \prime}$ et donc $f^{\prime}=f \operatorname{sur}\left(E_{r} \cap U_{p}\right) \times D_{p}^{\prime \prime}$, 
ce qui implique que $f^{\prime}=f \operatorname{sur}\left(E_{r} \cap U_{p}\right) \times D_{p}^{\prime}$ d'après le principe du prolongement analytique généralisé. D'autre part, $\widetilde{f}$ est holomorphe sur $U_{p} \times V_{p-1}$ et $\tilde{f}=f \operatorname{sur}\left(E_{r} \cap U_{p}\right) \times V_{p-1}$. Par conséquent $\tilde{f}=f^{\prime} \operatorname{sur}\left(E_{r} \cap U_{p}\right) \times D_{p}^{\prime} \cap V_{p-1}$, ce qui implique que $\widetilde{f}=f^{\prime}$ sur $U_{p} \times D_{p}^{\prime} \cap V_{p-1}$ d'après le principe du prolongement analytique généralisé. On obtient donc le prolongement holomorphe $f_{p}$ de $f$ à $U_{p} \times V_{p}$ en posant $f_{p}:=\widetilde{f}$ sur $U_{p} \times V_{p-1}$ et $f_{p}:=f^{\prime}$ sur $U_{p} \times D_{p}^{\prime}$, ce qui achève la récurrence. Pour $p=r$ on a alors $G \subset V_{r}$ et $f$ se prolonge en une application holomorphe de $U_{r} \times V_{r}$ dans $Z$, où $U_{r}$ est un voisinage de $a \in E_{r}^{*}$ et $V_{r}$ est un voisinage ouvert connexe de $G$.

Posons $E^{\prime}:=\bigcup_{r \geq s} E_{r}^{*}$. Alors $E^{\prime} \subset E^{*}$ est un borelien tel que $E \backslash E^{\prime} \subset$ $\bigcup_{r \geq s}\left(E_{r} \backslash E_{r}^{*}\right)$ soit pluripolaire et $f$ s'étend holomorphiquement au voisinage de chaque point de $E^{\prime} \times G$.

ÉtAPe 2. Considérons maintenant une suite croissante $\left(G_{j}\right)$ de compacts telle que $L=\bigcup_{j} G_{j}$. Alors la construction précédente fournit une suite $\left(E_{j}^{\prime}\right)$ d'ensembles boreliens contenus dans $E^{*}$ et tels que pour chaque $j \in \mathbb{N}^{*}$, l'ensemble $E \backslash E_{j}^{\prime}$ soit pluripolaire dans $X$ et l'application $f$ s'étende holomorphiquement au voisinage de chaque point de $E_{j}^{\prime} \times G_{j}$. En posant $E^{\prime}:=\bigcap_{j} E_{j}^{\prime}$, il en résulte que $E^{\prime} \subset E^{*}$ est un ensemble borelien tel que $E \backslash E^{\prime}=\bigcup_{j}\left(E \backslash E_{j}^{\prime}\right)$ soit pluripolaire dans $X$ et l'application $f$ s'étende holomorphiquement au voisinage de chaque point de $E^{\prime} \times L$.

4.2. Démonstration du théorème 2.2.1. La démonstration du théorème 2.2.1 se fait en trois étapes.

ÉtAPE 1. On suppose d'abord que $K \subset X$ et $L \subset Y$ sont des domaines de $X$ et $Y$ que l'on notera $U$ et $V$ respectivement. Ainsi $f$ est une application séparément holomorphe sur $(U \times F) \cup(E \times V)$ à valeurs dans $Z$.

Considérons une base dénombrable d'ouverts de $Z$ formée de domaines de Stein $\left(Z_{j}\right)_{j \geq 1}$ telle que pour chaque $j \geq 1$, il existe un plongement $\varphi_{j}$ de $Z_{j}$ sur un sous-ensemble analytique du polydisque unité de $\mathbb{C}^{\nu_{j}}$. Soient $\mathcal{D}=\left(D_{l}\right)$ et $\mathcal{G}=\left(G_{l}\right)$ des basees dénombrables d'ouverts de $X$ et $Y$ respectivement formées de domaines de Stein relativement compacts.

Soit $y_{0} \in F \cap F^{*}$ un point fixé. Alors pour tout $x \in E$ il existe $i=i_{x} \in \mathbb{N}^{*}$ tel que $f\left(x, y_{0}\right) \in Z_{i}$. En désignant, pour chaque $i \in \mathbb{N}^{*}$, par $A_{i}$ l'ensembles des $x \in E$ tels que $f_{x}\left(y_{0}\right):=f\left(x, y_{0}\right) \in Z_{i}$, on a alors $E=\bigcup_{i \geq 1} A_{i}$. Désignons par $I$ l'ensemble des indices $i \in \mathbb{N}^{*}$ tels que l'ensemble $A_{i}$ soit nonpluripolaire. Alors l'ensemble $A^{\prime}:=\bigcup_{i \in I} A_{i}$ est un sous-ensemble de $E$ quasiégal à $E$. Fixons $i \in I$; alors par définition, pour tout $x \in A_{i}$, il existe $j \in \mathbb{N}^{*}$ tel que $y_{0} \in G_{j}$ et $f_{x}\left(G_{j}\right) \subset Z_{i}$; on a utilisé la continuité de l'application partielle $f_{x}$ au point $y_{0} \in F \cap F^{*} \subset V$. Pour chaque $i \in I$ fixé, désignons par $B_{i, j}$ l'ensemble des $x \in A_{i}$ tels que $f_{x}\left(G_{j}\right) \subset Z_{i}$, de sorte que $A_{i}=\bigcup_{j \geq 1} B_{i, j}$ 
et $f\left(B_{i, j} \times G_{j}\right) \subset Z_{i}$. Notons, pour chaque $i \in I$, par $J_{i}$ l'ensemble des indices $j \in \mathbb{N}^{*}$ tels que l'ensemble $B_{i, j}$ soit non-pluripolaire. Alors l'ensemble $\widetilde{E}:=\bigcup_{i \in I} \bigcup_{j \in J_{i}} B_{i, j}$ est un sous-ensemble de $E$ quasi-égal à $E$.

Désormais fixons $i \in I$ et $j \in J_{i}$, et notons pour simplifier $B:=B_{i, j}$ et $G:=G_{j}$. Alors l'ensemble $M:=F \cap G$ est non-pluripolaire puisque $y_{0} \in F^{*}$ et que $G$ est un voisinage de $y_{0}$. Il en résulte que $B \times M \subset B \times G \subset W$ et que $f(B \times G) \subset Z_{i}$.

À partir de là, la démonstration de cette première étape se fait en deux sous-étapes.

1) Montrons d'abord que pour tout point $a \in B \cap B^{*}$, il existe un domaine $U_{a}^{\prime}$ voisinage de $a$ et un sous-ensemble borelien $E_{a}^{\prime} \subset U_{a}^{\prime} \cap B^{*}$ quasi-égal à $U_{a}^{\prime} \cap B$ tels que $f$ s'étende holomorphiquement au voisinage de chaque point de $E_{a}^{\prime} \times V$.

En effet, pour tout $y \in M=F \cap G$, on a $f^{y}(a)=f(a, y) \in Z_{i}$ et donc il existe $k \in \mathbb{N}^{*}$ tel que $a \in D_{k}$ et $f^{y}\left(D_{k}\right) \subset Z_{i}$. En raisonnant comme précédemment, on obtient un ensemble non-pluripolaire $N=N_{j, k} \subset M_{j}$ et un voisinage ouvert $D=D_{j_{k}} \Subset U$ de $a$ tels que $f(D \times N) \subset Z_{i}$. Posons $C:=B \cap D$ et $\Gamma:=(C \times G) \cup(D \times N) \subset W$. Alors par construction, on a $f(\Gamma) \subset Z_{i}$ et l'application $g:=\varphi \circ f: \Gamma \rightarrow \mathbb{C}^{\nu_{i}}$ est séparément holomorphe sur $\Gamma$, où $\varphi$ est un plongement holomorphe de $Z_{j}$ dans un polydisque de $\mathbb{C}^{\nu_{i}}$. Le théorème 3.4.2 implique alors que $g$ a une extension holomorphe $\widetilde{g}$ à l'ouvert

$$
\widehat{\Gamma}:=\{(x, y) \in D \times G: \omega(x ; C ; D)+\omega(y ; N ; G)<1\} .
$$

Le principe du prolongement analytique généralisé prouve que $\widetilde{g}(\widehat{\Gamma}) \subset \varphi\left(Z_{i}\right)$. En observant que $\widehat{\Gamma}=\bigcup_{k \geq 1}\left(O_{k} \times P_{k}\right)$, où

$$
\begin{aligned}
O_{k} & :=\{x \in D: \omega(x ; C ; D)<1-1 / k\} \\
P_{k} & :=\{y \in G: \omega(y ; N ; G)<1 / k\},
\end{aligned}
$$

on en déduit que $f$ est holomorphe sur chaque rectangle ouvert $O_{k} \times P_{k}$ et que pour tout $x \in C \cap O_{k}$, qui est non-pluripolaire, l'application partielle $f(x, \cdot)$ se prolonge holomorphiquement à $U$. Grâce au lemme 4.1.1, on en déduit qu'il existe un sous-ensemble borelien $C_{k}^{\prime} \subset O_{k} \cap C^{*}$ quasi-égal à $C \cap O_{k}$ tel que $f$ admette une extension holomorphe au voisinage de chaque point de $C_{k}^{\prime} \times V$. Il en résulte immédiatement que $C^{\prime}:=\bigcup_{k} C_{k}^{\prime}$ est un ensemble borelien quasi-égal à $C=B \cap D$, tel que $C^{\prime} \subset C^{*}=D \cap B^{*}$ et tel que $f$ admette une extension holomorphe au voisinage de chaque point de $C^{\prime} \times V$. En posant $E_{a}^{\prime}:=C^{\prime}$ et $U_{a}:=D$, on obtient le résultat annoncé.

2) Montrons maintenant qu'il existe un sous-ensemble borelien $E^{\prime} \subset E^{*}$ quasi-égal à $E$ tel que l'application $f$ admette une extension holomorphe au voisinage de chaque point de $E^{\prime} \times V$. 
En effet, en recouvrant $B \cap B^{*}$ par une famille dénombrable de voisinages du type $U_{a}^{\prime}, a \in T$, où $T$ est un ensemble dénombrable, et en considérant les ensembles boreliens correspondants $E_{a}^{\prime} \subset B^{*} \cap U_{a}^{\prime}$ quasi-égaux à $B \cap U_{a}^{\prime}$, obtenus à l'étape précédente, on en déduit immédiatement que l'ensemble $E^{\prime \prime}:=\bigcup_{a \in T} E_{a}^{\prime}$ est un borelien tel que $E^{\prime \prime} \subset B^{*}$ soit quasi-égal à $B$ et que $f$ admette une extension holomorphe au voisinage de chaque point de $E^{\prime \prime} \times V$.

Rappelons à ce stade que $B=B_{i, j} \subset E$ avec $i \in I$ et $j \in J_{i}$ et notons par $E_{i, j}^{\prime \prime} \subset B_{i, j}^{*}$ l'ensemble borelien correspondant quasi-égal à $B_{i, j}$ et tel que $f$ admette une extension holomorphe au voisinage de chaque point de $E_{i, j}^{\prime \prime} \times V$. Posons $E^{\prime}:=\bigcup_{i \in I} \bigcup_{j \in J_{i}} E_{i, j}^{\prime \prime}$. Alors l'ensemble $E^{\prime} \subset E^{*}$ est un borelien quasi-égal à l'ensemble $\widetilde{E}=\bigcup_{i \in I} \bigcup_{j \in J_{i}} B_{i, j}$, qui est quasi-égal à $E$, d'après le début de la preuve. Par conséquent l'ensemble $E^{\prime} \subset E^{*}$ est un borelien quasi-égal à $E$ et tel que $f$ admette une extension holomorphe au voisinage de chaque point de $E^{\prime} \times V$.

Comme les deux facteurs jouent des rôles symétriques dans l'énoncé du théorème, le résultat annoncé en découle.

ÉtAPE 2. On suppose que $K \subset X$ et $L \subset Y$ sont des compacts. Soit $\left(D_{j}\right)$ (resp. $\left(G_{j}\right)$ ) une suite de domaines de $X$ (resp. $Y$ ) qui converge vers $K\left(\right.$ resp. $L$ ). Pour chaque $j \in \mathbb{N}$, désignons par $E_{j}$ (resp. $F_{j}$ ) l'ensemble des $x \in E$ (resp. $y \in F$ ) tels que $f(x, \cdot)$ (resp. $f(\cdot, y)$ ) se prolonge en une fonction holomorphe sur $G_{j}$ (resp. $D_{j}$ ). Il est alors clair que $E=\bigcup_{j} E_{j}$ et $F=\bigcup_{j} F_{j}$. Il existe donc un rang $j_{0}>0$ tel que pour $j \geq j_{0}$, les ensembles $E_{j}$ et $F_{j}$ soient non-pluripolaires dans $X$ et $Y$ respectivement. De plus pour chaque $j \geq j_{0}$, on peut donc définir une fonction $f_{j}: W_{j}:=\left(E_{j} \times G_{j}\right) \cup\left(D_{j} \times F_{j}\right) \rightarrow \mathbb{C}$ séparément holomorphe telle que $f_{j}=f$ sur $\left(E_{j} \times L\right) \cup\left(K \times F_{j}\right)$. D'après la première étape, pour chaque $j \geq j_{0}$, il existe un ensemble borelien $E_{j}^{\prime} \subset E_{j}^{*}$ quasi-égal à $E_{j}$ et un ensemble borelien $F_{j}^{\prime} \subset F_{j}$ quasi-égal à $F_{j}$ tels que l'application $f_{j}$ s'étend holomorphiquement au voisinage de chaque point de $\left(D_{j} \times F_{j}^{\prime}\right) \cup\left(E_{j}^{\prime} \times G_{j}\right)$. Il en résulte que $f$ s'étend holomorphiquement au voisinage de chaque point de $\left(K \times F_{j}^{\prime}\right) \cup\left(E_{j}^{\prime} \times L\right)$.

Posons $E^{\prime}:=\bigcup_{j \geq j_{0}} E_{j}^{\prime}$ et $F^{\prime}:=\bigcup_{j \geq j_{0}} F_{j}^{\prime}$. Alors il en résulte que $E^{\prime} \subset E^{*}$ est un ensemble borelien quasi-égal à $E$ et que $F^{\prime}$ est un ensemble borelien quasi-égal à $F$ tels que que $f$ se prolonge holomorphiquement au voisinage de chaque point de $\left(K \times F^{\prime}\right) \cup\left(E^{\prime} \times L\right)$.

ÉTAPE 3. Passons maintenant au cas général. On écrit alors $K=\bigcup_{j} K_{j}$ et $L=\bigcup_{j} L_{j}$, où $\left(K_{j}\right)$ et $\left(L_{j}\right)$ forment des suites croissantes d'ensembles compacts et non-pluripolaires dans $X$ et $Y$ respectivement. En posant $E_{j}:=$ $E \cap K_{j}$ et $F_{j}:=F \cap L_{j}$, on voit clairement que pour $j$ assez grand $E_{j}$ et $F_{j}$ sont non-pluripolaires dans $X$ et $Y$ respectivement. D'après la deuxième étape, pour chaque $j$ assez grand, il existe un ensemble borelien $E_{j}^{\prime} \subset E_{j}^{*}$ quasi-égal à $E_{j}$ et un ensemble borelien $F_{j}^{\prime} \subset F_{j}^{*}$ quasi-égal à $F_{j}$ tels que 
$f$ se prolonge holomorphiquement au voisinage de chaque point de $\left(K_{j} \times\right.$ $\left.F_{j}^{\prime}\right) \cup\left(E_{j}^{\prime} \times L_{j}\right)$. Posons $E^{\prime}:=\bigcap_{j} E_{j}^{\prime}$ et $F^{\prime}:=\bigcap_{j} F_{j}^{\prime}$. Il est facile de voir que $E^{\prime} \subset E^{*}$ est un ensemble borelien quasi-égal à $E$ et que $F^{\prime} \subset F^{*}$ est un ensemble borelien quasi-égal à $F$ tels que $f$ s'étende holomorphiquement au voisinage de chaque point de $\left(K_{j} \times F^{\prime}\right) \cup\left(E^{\prime} \times L_{j}\right)$ pour tout $j \in \mathbb{N}^{*}$. Par suite l'application $f$ s'étend holomorphiquement au voisinage de chaque point de $\left(K \times F^{\prime}\right) \cup\left(E^{\prime} \times L\right)$. Pour achever la démonstration du théorème, posons $S_{1}:=E^{*} \backslash E^{\prime} \subset X$ et $S_{2}:=F^{*} \backslash F^{\prime} \subset Y$. Alors, par construction, les ensembles $S_{1}$ et $S_{2}$ sont pluripolaires dans $X$ et $Y$ respectivement et d'après ce qui précède, on a $S(f, W) \subset S_{1} \times S_{2}$, ce qui prouve par définition que $S(f, W)$ est doublement pluripolaire dans $X \times Y$.

4.3. Démonstration du théorème 2.2.2. Pour démontrer le théorème 2.2.2, on distinguera deux cas.

Premier Cas. On suppose que $K=U \subset X$ et $L=V \subset Y$ sont des ouverts. Soit $f$ est une application séparément holomorphe sur $W=$ $(U \times F) \cup(E \times V)$ à valeurs dans $Z$. D'après le théorème 2.2.1, il existe des ensembles boreliens $A \subset E^{*}$ et $B \subset F^{*}$ quasi-égaux à $E$ et $F$ respectivement tels que $f$ s'étende holomorphiquement au voisinage de chaque point de $W^{\prime}:=(U \times B) \cup(A \times V)$. D'après la proposition 3.4.3, il existe des ensembles boreliens $A^{\prime} \subset A^{*}$ et $B^{\prime} \subset B^{*}$ quasi-égaux à $A$ et $B$ respectivement, et il existe un voisinage ouvert $\Omega$ de $\left(U \times B^{\prime}\right) \cup\left(A^{\prime} \times V\right)$ tels que $f$ s'étende en une application holomorphe de $\Omega$ dans $Z$. Il est alors clair que $A^{\prime} \subset E^{*}$ et $B^{\prime} \subset F^{*}$ sont des ensembles boreliens quasi-égaux à $E$ et $F$ respectivement, ce qui achève la démonstration du théorème 2.2.2 dans le premier cas.

Pour démontrer le théorème 2.2 .2 dans le second cas, on aura besoin d'étendre la proposition 3.4.3 au cas d'une croix inscrite dans un rectangle de type $\mathcal{G}_{\delta}^{s}$. Cela utilise le premier cas du théorème 2.2.2 que l'on vient d'établir. Voici le résultat intermédiaire dont nous aurons besoin.

Proposition 4.3.1. Soient $X, Y, Z$ des espaces analytiques complexes et $K \subset X, L \subset Y$ des ensembles boreliens connexes non-pluripolaires de type $\mathcal{G}_{\delta}^{s}$. Soient $A \subset K, B \subset L$ des ensembles non-pluripolaires et $f: \Sigma:=$ $(K \times B) \cup(A \times L) \rightarrow Z$ une application qui s'étend holomorphiquement au voisinage de chaque point de $\Sigma$. Alors il existe un ensemble borelien $A^{\prime} \subset A^{*}$ quasi-égal à $A$ dans $X$, un ensemble borelien $B^{\prime} \subset B^{*}$ quasi-égal à $B$ dans $Y$, un voisinage ouvert $\Omega$ de la croix $\Sigma^{\prime}:=\left(K \times B^{\prime}\right) \cup\left(A^{\prime} \times L\right)$ et une application holomorphe $\widetilde{f}: \Omega \rightarrow Z$ telle que $\widetilde{f}=f$ sur $\Omega \cap \Sigma$.

Démonstration. La démonstration est analogue à celle de la proposition 3.4.3 avec cependant une perte d'information concernant les ensembles $A^{\prime}$ et $B^{\prime}$. En effet, soit $\left(D_{j}\right)$ (resp. $\left.\left(G_{j}\right)\right)$ une suite décroissante de domaines de $X$ (resp. $Y$ ) qui converge vers $K$ (resp. $L$ ). 
Alors pour chaque entier $j \geq 1$, désignons par $A_{j}$ (resp. $B_{j}$ ) l'ensemble des points $x \in A$ (resp. $y \in B$ ) tels que l'application partielle $f(x, \cdot)$ (resp. $f(\cdot, y))$ se prolonge en une application holomorphe de $G_{j-1}\left(\operatorname{resp} . D_{j-1}\right.$ ) dans $Z$. Il en résulte immédiatement que $A=\bigcup_{j} A_{j}$ et $B=\bigcup_{j} B_{j}$, de sorte qu'il existe un $j_{0} \geq 1$ tel que $A_{j}$ et $B_{j}$ soient non-pluripolaires pour tout $j \geq j_{0}$, puisque les suites d'ensembles en question sont croissantes. De plus on obtient une application séparément holomorphe $f_{j}$ sur $\Sigma_{j}:=$ $\left(D_{j} \times B_{j}\right) \cup\left(A_{j} \times G_{j}\right)$ telle que $f_{j}=f$ sur $\left(K \times B_{j}\right) \cup\left(A_{j} \times L\right)$.

Fixons $j \geq j_{0}$. D'après le premier cas du théorème 2.2.2, il existe des ensembles boreliens $A_{j}^{\prime} \subset A_{j}^{*}$ et $B_{j}^{\prime} \subset B_{j}^{*}$ quasi-égaux à $A_{j}$ et $B_{j}$ respectivement tel que l'application $f_{j}$ s'étend holomorphiquement au voisinage de $\Sigma_{j}^{\prime}:=\left(A_{j}^{\prime} \times G_{j}\right) \cup\left(D_{j} \times B_{j}^{\prime}\right)$. On est donc dans les hypothèses de la proposition 3.4.3. Comme $D_{j} \Subset D_{j-1}$ et $G_{j} \Subset G_{j-1}$, d'après la première étape de la démonstration de la proposition 3.4.3, il existe un voisinage consistant $D_{j}^{\prime} \subset D_{j}$ de $A_{j}^{\prime \prime}:=A_{j}^{\prime} \cap A_{j}^{\prime *}$, un voisinage consistant $G_{j}^{\prime} \subset G_{j}$ de $B_{j}^{\prime \prime}:=B_{j}^{\prime} \cap B_{j}^{\prime *}$ et une application $h_{j}$ holomorphe sur l'ouvert $\Omega_{j}:=\left(D_{j}^{\prime} \times G_{j}\right) \cup\left(D_{j} \times G_{j}^{\prime}\right)$ telle que $h_{j}=f_{j}$ sur $\left(D_{j}^{\prime} \times B_{j}^{\prime \prime}\right) \cup\left(A_{j}^{\prime \prime} \times G_{j}\right)$. En particulier on a $h_{j}=f \operatorname{sur}\left(K \times B_{j}^{\prime \prime}\right) \cup\left(A_{j}^{\prime \prime} \times L\right)$.

Montrons que les applications $h_{j}$ se recollent. En effet, fixons deux indices $j \geq i \geq j_{0}$ et observons que

$$
\Omega_{j} \cap \Omega_{i}=R_{1} \cup R_{2} \cup R_{3} \cup R_{4}
$$

où

$$
\begin{array}{ll}
R_{1}:=\left(D_{i}^{\prime} \cap D_{j}^{\prime}\right) \times G_{j}, & R_{2}:=\left(D_{i}^{\prime} \cap D_{j}\right) \times G_{j}^{\prime}, \\
R_{3}:=D_{j}^{\prime} \times\left(G_{i}^{\prime} \cap G_{j}\right), & R_{4}:=D_{j} \times\left(G_{i}^{\prime} \cap G_{j}^{\prime}\right) .
\end{array}
$$

On constate comme à l'étape 2 que chaque rectangle $R$ parmi les rectangles $R_{i}(1 \leq i \leq 4)$ contient l'ensemble $A_{j}^{\prime \prime} \times B_{j}^{\prime \prime}$ et que l'une des deux projections de $R$ est un voisinage consistant de la projection correspondante de l'ensemble $A_{j}^{\prime \prime} \times B_{j}^{\prime \prime}$. On peut donc raisonner comme dans la démonstration de la proposition 3.4.3 pour conclure, grâce au principe du prolongement analytique généralisé, que $h_{i}=h_{j}$ sur $\Omega_{j} \cap \Omega_{i}$, ce qui prouve qu'il existe une application $h$ holomorphe sur $\Omega:=\bigcup_{j} \Omega_{j}$ à valeurs dans $Z$ telle que $h=h_{j}$ $\operatorname{sur} \Omega_{j}$ pour tout $j \geq j_{0}$.

De plus il est clair que $h$ est une extension holomorphe de $f$ à $\Omega$. D'autre part, l'ensemble $A^{\prime}:=\bigcup A_{j}^{\prime \prime}$ est un borelien contenu dans $A^{*}$ et quasi-égal à $A$; de même que l'ensemble $B^{\prime}:=\bigcup B_{j}^{\prime \prime}$ est un ensemble borelien contenu dans $B^{*}$ et quasi-égal à $B$. De plus $\Omega$ est un voisinage de $\Sigma^{\prime}:=\left(K \times B^{\prime}\right) \cup$ $\left(A^{\prime} \times L\right)$.

Nous sommes maintenant en mesure de démontrer le théorème 2.2.2 dans le second cas. 
Second Cas. On suppose que $K \subset X$ et $L \subset Y$ sont des ensembles de type $\mathcal{G}_{\delta}^{s}$. Soit $f$ est une application séparément holomorphe sur $W=(K \times F) \cup(E \times L)$ à valeurs dans $Z$. D'après le théorème 2.2.1, il existe des ensembles boreliens $A \subset E^{*}$ et $B \subset F^{*}$ quasi-égaux à $E$ et $F$ respectivement tels que $f$ s'étende holomorphiquement au voisinage de chaque point de la croix $W^{\prime}:=(K \times B) \cup(A \times L)$. D'après la proposition 4.3.1, il existe des ensembles boreliens $A^{\prime} \subset A^{*}$ et $B^{\prime} \subset B^{*}$ quasi-égaux à $A$ et $B$ respectivement, et il existe un voisinage ouvert $\Omega$ de $\left(K \times B^{\prime}\right) \cup\left(A^{\prime} \times B\right)$ tels que $f$ s'étende en une application holomorphe de $\Omega$ dans $Z$. Il est alors clair que $A^{\prime} \subset E^{*}$ et $B^{\prime} \subset F^{*}$ sont des ensembles boreliens quasi-égaux à $E$ et $F$ respectivement, ce qui achève la démonstration du théorème 2.2.2.

4.4. Démonstration du théorème 2.2.4 et du corollaire 2.2.5. Nous allons maintenant utiliser les résultats précédents pour démontrer le théorème 2.2.4 et le corollaire 2.2.5.

Démonstration du théorème 2.2.4. 1) Supposons d'abord que $X$ et $Y$ sont des variétés de Stein. Alors le théorème 2.2.4 est une conséquence immédiate du théorème 3.4.2 et du théorème 2.1.2 de Shiffman.

2) Supposons maintenant que $X$ et $Y$ sont des variétés complexes. Dans ce cas, la démonstration se fait en deux étapes.

ÉtAPe 1. On suppose que $U \subset X$ est un domaine de Stein, $E \subset U$ est un ensemble non-pluripolaire, $F=V$ est un domaine quelconque de $Y$. Nous allons montrer que si $f: W=W(E \times V ; U \times V) \rightarrow Z$ est séparément holomorphe sur $W$ alors elle est holomorphe sur $U \times V$.

En effet, soit $G \Subset V$ un domaine de Stein quelconque. Alors la restriction $g$ de $f$ à la croix $W(E \times G ; U \times G)=U \times G$ est séparément holomorphe. Comme elle est inscrite dans le rectangle $U \times G$, qui est une variété de Stein produit, alors d'après le premier cas $g$ s'étend en une application holomorphe $\widetilde{g}$ sur l'enveloppe d'holomorphie de $W(E \times G ; U \times G)$, qui n'est autre que le domaine de Stein $U \times G$. Il en résulte que $f$ est holomorphe sur $U \times G$ et comme $G \subset V$ est un domaine de Stein quelconque, on en déduit que $f$ est holomorphe sur $U \times V$.

Étape 2. Dans le cas général, on va utiliser un résultat de J. E. Fornæss et E. Stout pour se ramener au premier cas et ensuite utiliser le résultat de la première étape pour conclure grâce à la proposition 3.4.3.

En effet, d'après [Fo-St], si $m$ est la dimension de $X$, il existe un biholomorphisme local $\phi: \Delta^{m} \rightarrow X$ du polydisque unité $\Delta^{m}$ de $\mathbb{C}^{m}$ sur $X$. De même il existe un biholomorphisme local $\psi: \Delta^{n} \rightarrow Y$, où $n$ est la dimension de $Y$. Posons $D:=\phi^{-1}(U), A:=\phi^{-1}(E), G:=\psi^{-1}(V), B:=\psi^{-1}(F)$. Alors l'application définie par $h(z, w):=f(\phi(z), \psi(w))$ est séparément holomorphe sur la croix $\Sigma:=(D \times B) \cup(A \times G) \subset \Delta^{m} \times \Delta^{n}$ à valeurs dans 
$Z$. D'après le premier cas, $h$ s'étend en une application $\widetilde{h}$ holomorphe sur l'enveloppe d'holomorphie $\widetilde{\Sigma}$ de $\Sigma$ à valeurs dans $Z$. Il est facile de vérifier que $A^{*}=\phi^{-1}\left(E^{*}\right)$ et $B^{*}=\psi^{-1}\left(F^{*}\right)$.

Montrons que $f$ s'étend holomorphiquement au voisinage de chaque point de $W^{*}$. En effet, soient $\left(x_{0}, y_{0}\right) \in E^{*} \times L$ et $\left(z_{0}, w_{0}\right) \in A^{*} \times G$ tels que $\phi\left(z_{0}\right)=x_{0}$ et $\psi\left(w_{0}\right)=y_{0}$. Comme $\left(z_{0}, w_{0}\right) \in \widetilde{\Sigma}$, il existe alors un domaine $D_{0} \subset \Delta^{m}$ voisinage de $z_{0}$ et un domaine $G_{0} \subset \Delta^{n}$ voisinage de $w_{0}$ tel que $G_{0} \cap F^{*} \neq \emptyset$ et que $D_{0} \times G_{0} \subset \widetilde{\Sigma}$. Par suite l'application $\widetilde{h}$ est holomorphe sur $D_{0} \times G_{0}$. On peut toujours choisir $D_{0}$ assez petit pour que $\phi$ induise un biholomorphisme de $D_{0}$ sur le domaine $U_{0}:=\phi\left(D_{0}\right)$. Alors pour chaque $y \in$ $\psi\left(G_{0}\right)$, en prenant $\zeta \in G_{0}$ tel que $\psi(\zeta)=y$, on en déduit que $f(\phi(z), y)=$ $\widetilde{h}(z, \zeta)$ pour tout $z \in D_{0} \cap A^{*}$. Comme $\phi$ est un biholomorphisme de $D_{0}$ sur $U_{0}$, il en résulte que l'application $x \mapsto f^{y}(x):=f(x, y)$, définie sur $\phi\left(D_{0} \cap A^{*}\right)=U_{0} \cap E^{*}$, se prolonge en une application $\widetilde{f^{y}}$ holomorphe sur $U_{0}$. D'autre part, si $x \in U_{0} \cap E^{*}$, l'application $y \mapsto f(x, y)$ est holomorphe sur $G_{0}$. Ainsi l'application $g:=\left(\left(U_{0} \cap E^{*}\right) \times V_{0}\right) \cup\left(U_{0} \times V_{0}\right) \rightarrow Z$ définie $\operatorname{par} g(x, y):=\widetilde{f^{y}}(x)$ pour $(x, y) \in U_{0} \times V_{0}$ est séparément holomorphe sur la croix $\left(\left(U_{0} \cap E^{*}\right) \times V_{0}\right) \cup\left(U_{0} \times V_{0}\right)$ à valeurs dans $Z$. Maintenant nous sommes ramenés à la première étape, ce qui implique que $g$ est holomorphe sur $U_{0} \times V_{0}$. Par conséquent, l'application $f$ s'étend holomorphiquement à l'ouvert $U_{0} \times V_{0}$, qui est un voisinage admissible du point $\left(x_{0}, y_{0}\right)$. Ainsi l'application $f$ s'étend holomorphiquement à un voisinage admissible de chaque point de $E^{*} \times V$. Par symétrie, il en résulte que $f$ s'étend holomorphiquement à un voisinage de chaque point de $W^{*}$. On conclut alors en utilisant la proposition 3.4.3.

\section{Il nous reste maintenant à démontrer le corollaire 2.2.5.}

Démonstration du corollaire 2.2.5. Soit $\left(G_{j}\right)_{j}$ une suite décroissante de domaines de $Y$ qui converge vers $L$. Notons $E_{j}$ l'ensemble des $x \in E$ tels que l'application $f_{x}: L \rightarrow Z$ se prolonge en une application holomorphe $g_{x, j}$ sur l'ouvert $G_{j}$. Alors $\left(E_{j}\right)$ est une suite croissante d'ensembles tels que $E=\bigcup_{j} E_{j}$. Il en résulte que pour tout $j$ assez grand, $E_{j}$ est non-pluripolaire. Par conséquent, l'application $f_{j}: W_{j}:=W\left(E_{j} \times G_{j} ; U \times L\right) \rightarrow Z$, donnée $\operatorname{par} f_{j}(x, y):=g_{x, j}(y)$ pour $(x, y) \in E_{j} \times G_{j}$ et $f_{j}(x, y):=f(x, y)$ pour $(x, y) \in U \times L$, est bien définie et est séparément holomorphe sur $W_{j}$. D'après le théorème 2.2.3, l'application $f_{j}$ s'étend holomorphiquement à un voisinage ouvert $\Omega_{j}$ de $W_{j}^{*}=\left(E_{j}^{*} \times G_{j}\right) \cup\left(U \times L^{*}\right)$, qui contient $U \times L^{*}$.

Supposons de plus que $U$ est hyperconvexe et posons $A:=\bigcup_{j} E_{j}^{*}$. Notons que $E \backslash A \subset \bigcup_{j}\left(E_{j} \backslash E_{j}^{*}\right)$ est pluripolaire dans $U$ et $\operatorname{donc} \operatorname{Cap}^{*}(E \backslash A ; U)=0$. Comme $E \backslash A=\bigcap_{j}\left(E \backslash E_{j}^{*}\right)$ et que la suite $\left(E \backslash E_{j}^{*}\right)$ est décroissante, il en résulte que pour $\varepsilon>0$ donné, on peut trouver $j$ assez grand tel que 
$\operatorname{Cap}^{*}\left(E \backslash E_{j}^{*} ; U\right)<\varepsilon$. D'après ce qui précède, $f$ s'étend holomorphiquement à l'ouvert $\Omega_{j}$, voisinage de $\left(E_{j}^{*} \times G_{j}\right) \cup\left(U \times L^{*}\right)$. En posant $E^{\prime}:=E_{j}^{*}$ et $G:=G_{j}$, on obtient le résultat souhaité.

Remerciements. Ce travail a bénéficié d'un large soutien dans le cadre de l'Action Integrée Franco-Marocaine d'Analyse Complexe et Géométrie (no. 180MA99) ainsi que du Programme d'Aide à la Recherche Scientifique (PARS) financé par la Direction Marocaine de la Recherche Scientifique. Nous tenons à remercier les institutions concernées pour leur aide ainsi que le Professeur Bensalem Jennane de la Faculté des Sciences de Rabat, responsable scientifique de ces programmes, pour son accueil chaleureux lors de nos différents séjours à Rabat.

\section{Références}

[A-S-Y] K. Adachi, M. Suzuki and M. Yoshida, Continuation of holomorphic mappings with values in a complex Lie group, Pacific J. Math. 47 (1973), 1-4.

[Al1] O. Alehyane, Une extension du théorème de Hartogs pour les applications séparément holomorphes, C. R. Acad. Sci. Paris Sér. I 324 (1997), 149-152.

[Al2] - Applications séparément méromorphes dans les espaces analytiques, prépublication du Laboratoire E. Picard, UFR MIG, Univ. Paul Sabatier, Toulouse no. 79 (1996).

[Al-He] O. Alehyane et J. M. Hecart, Propriétés de stabilité de la fonction extrémale relative, préprint, 1999.

[Bed] E. Bedford, The operator $\left(d d^{c}\right)^{n}$ on complex spaces, dans : Séminaire d'analyse Lelong-Skoda, Lecture Notes in Math. 919, Springer, 1981, 294-324.

[Be-Ta] E. Bedford and B. A. Taylor, A new capacity for plurisubharmonic functions, Acta Math. 149 (1982), 1-40.

[Ber] S. Bergman, The Kernel Function and Conformal Mapping, Math. Surveys 5, Amer. Math. Soc., 1950.

[Bl] Z. Błocki, Singular sets of separately analytic functions, Ann. Polon. Math. 56 (1992), 219-225.

[Bo] H. J. Borchers, The generalized three circle and other convexity theorems with applications to the construction of envelopes of holomorphy, Ann. Inst. H. Poincaré 27 (1977), 31-60.

[De] J. P. Demailly, Mesures de Monge-Ampère et caractérisation géométrique des variétés algébriques affines, Mém. Soc. Math. France 19 (1985).

[Fo-St] J. E. Fornæss and E. L. Stout, Spreading polydiscs on complex manifolds, Amer. J. Math. 99 (1977), 933-960.

[Ha] F. Hartogs, Zur Theorie der analytischen Funktionen mehrer unabhängiger Veränderlichen, insbesondere über die Darstellung derselben durch Reihen, welche nach Potenzen einer Veränderlichen fortschreiten, Math. Ann. 62 (1906), 1-88.

[Iv1] S. M. Ivashkovich, The Hartogs phenomenon for holomorphically convex Kähler manifolds, Math. USSR-Izv. 29 (1987), 225-232.

[Iv2] - An example concerning extension and separate analyticity properties of meromorphic mappings, Amer. J. Math. 121 (1999), 97-130. 
[Ja] W. Jarnicki, communication personnelle.

[Kl] M. Klimek, Pluripotential Theory, Oxford Univ. Press, 1991.

[Mi] B. Mityagin, Approximate dimension and bases in nuclear spaces, Russian Math. Surveys 16 (1961), 59-127.

[Ng] Nguyen Thanh Van, Note on doubly orthogonal systems of Bergman, Linear Topol. Spaces Complex Anal. 3 (1997), 157-159.

[Ng-Ze1] Nguyen Thanh Van et A. Zeriahi, Familles de polynômes presque partout bornées, Bull. Sci. Math. 107 (1983), 81-91.

[Ng-Ze2] - - - Une extension du théorème de Hartogs sur les fonctions séparément analytiques, dans : Analyse Complexe Multivariable : Récents Développements, (Guadeloupe, 1988), Alex Meril (éd.), EditEl, Rende, 1991, 183-194.

[Ng-Ze3] -, 一, Systèmes doublement orthogonaux de fonctions holomorphes et applications, dans : Topics in Complex Analysis, Banach Center Publ. 31, Inst. Math., Polish Acad. Sci., Warszawa, 1995, 281-297.

[Ku-Ha] Nguyen Van Khue and Lee Mau Hai, Separately holomorphic mappings on compact subsets, preprint of the Pedagogical Institute of Hanoi, 1999.

[Ra] J. Saint Raymond, Fonctions séparément analytiques, Ann. Inst. Fourier (Grenoble) 40 (1990), 79-101.

[Sa] A. Sadullaev, Plurisubharmonic measures and capacities on complex manifolds, Russian Math. Surveys 36 (1981), 61-119.

[Shif1] B. Shiffman, Extension of holomorphic maps into Hermitian manifolds, Math. Ann. 194 (1971), 249-258.

[Shif2] -, On separate analyticity and Hartogs theorem, Indiana Univ. Math. J. 38 (1989), 943-957.

[Shif3] - Hartogs theorem for separately holomorphic mappings into complex spaces, C. R. Acad. Sci. Paris 310 (1990), 89-94.

[Shim] I. Shimoda, Notes on the functions of two complex variables, J. Gakugei Tokushima Univ. 8 (1957), 1-3.

[Si1] J. Siciak, Analyticity and separate analyticity of functions defined on lower dimensional subsets of $\mathbb{C}^{n}$, Zeszyty Nauk. Univ. Jagielloń. Prace Mat. 13 (1969), $53-70$.

[Si2] - Separately analytic functions and envelopes of holomorphy of some lower dimensional subsets of $\mathbb{C}^{n}$, Ann. Polon. Math. 22 (1970), 145-171.

[Si3] - Extremal plurisubharmonic functions in $\mathbb{C}^{n}$, ibid. 34 (1981), 175-211.

[Si4] - Singular sets of separately analytic functions, Colloq. Math. 60-61 (1990), 281-290.

[Te] T. Terada, Sur une certaine condition sous laquelle une fonction de plusieurs variables complexes est holomorphe, Publ. Res. Inst. Math. Sci. Ser. A 2 (1967), 383-396.

[W] H. Wu, Normal families of holomorphic mappings, Acta Math. 119 (1967), 193-233.

[Za1] V. P. Zahariuta, Fonctions plurisousharmoniques extrémales, échelles hilbertiennes et isomorphismes d'espaces de fonctions analytiques de plusieurs variables, I, II, Teor. Funktsiü Funktsional. Anal. i Prilozhen. 19 (1974), 133-157 et 21 (1974), 65-83 (en russe).

[Za2] - Separately holomorphic functions, generalisations of Hartogs theorem and envelopes of holomorphy, Math. USSR-Sb. 30 (1976), 51-76.

[Ze1] A. Zeriahi, Bases de Schauder et isomorphismes d'espaces de fonctions holomorphes, C. R. Acad. Sci. Paris 310 (1990), 691-694. 
[Ze2] A. Zeriahi, Fonction de Green pluricomplexe à pôle à l'infini sur un espace de Stein parabolique et applications, Math. Scand. 69 (1991), 89-126.

[Ze3] -, Comportement asymptotique des systèmes doublement orthogonaux de Bergman : Une approche élémentaire, préprint du Laboratoire de Mathématiques Emile Picard de l'UPS-Toulouse, 2000.

Département de Mathématiques

Université Chouaib Doukkali

Faculté des Sciences, B.P. 20

El Jadida, Maroc

E-mail: alehyane@ucd.ac.ma
Laboratoire de Mathématiques Emile Picard UMR-CNRS 5580

Université Paul Sabatier 118 route de Narbonne F-31062 Toulouse, France E-mail: zeriahi@picard.ups-tlse.fr

Reçu par la Rédaction le 5.5.2000

Révisé le 26.2.2001 